\title{
Equality tests of high-dimensional covariance matrices under the strongly spiked eigenvalue model
}

\author{
Aki Ishii ${ }^{\mathrm{a}}$, Kazuyoshi Yata ${ }^{\mathrm{b}}$, Makoto Aoshima ${ }^{\mathrm{b}, 1}$ \\ ${ }^{a}$ Department of Information Sciences, Tokyo University of Science, Chiba, Japan \\ ${ }^{b}$ Institute of Mathematics, University of Tsukuba, Ibaraki, Japan
}

\begin{abstract}
We consider the equality test of high-dimensional covariance matrices under the strongly spiked eigenvalue (SSE) model. We find the difference of covariance matrices by dividing high-dimensional eigenspaces into the first eigenspace and the others. We create a new test procedure on the basis of those high-dimensional eigenstructures. We precisely study the influence of spiked eigenvalues on a test statistic and consider its bias correction so that the proposed test procedure has a consistency property for the size. We also show that the proposed test procedure has preferable properties for the power. We discuss the performance of the test procedure by simulations. We give a demonstration in actual data analyses using microarray data sets.
\end{abstract}

Keywords: HDLSS, Large $p$ small $n$, Noise-reduction methodology, SSE model, Two-sample test

2000 MSC: primary $62 \mathrm{H} 15$, secondary $62 \mathrm{H} 25$

\section{Introduction}

In this paper, we consider the equality test of covariance matrices when the data dimension is much larger than the sample size. Suppose we have two classes $\pi_{i}, i=1,2$. We define independent $d \times n_{i}$ data matrices, $\boldsymbol{X}_{i}=\left[\boldsymbol{x}_{i 1}, \ldots, \boldsymbol{x}_{i n_{i}}\right], i=$ 1,2 , for $\pi_{i}, i=1,2$. We assume that $\boldsymbol{x}_{i j}, j=1, \ldots, n_{i}$, are independent and identically distributed (i.i.d.) as a $d$-dimensional distribution with a mean vector $\boldsymbol{\mu}_{i}$

Email address: aoshima@math.tsukuba . ac.jp (Makoto Aoshima)

${ }^{1}$ Institute of Mathematics, University of Tsukuba, Ibaraki 305-8571, Japan;

Fax: +81-298-53-6501 
and covariance matrix $\boldsymbol{\Sigma}_{i}$. We assume $n_{i} \geq 4, i=1,2$. The eigen-decomposition of $\boldsymbol{\Sigma}_{i}$ is given by $\boldsymbol{\Sigma}_{i}=\boldsymbol{H}_{i} \boldsymbol{\Lambda}_{i} \boldsymbol{H}_{i}^{T}$, where $\boldsymbol{\Lambda}_{i}=\operatorname{diag}\left(\lambda_{1(i)}, \ldots, \lambda_{d(i)}\right)$ having $\lambda_{1(i)} \geq \cdots \geq \lambda_{d(i)}(\geq 0)$ and $\boldsymbol{H}_{i}=\left[\boldsymbol{h}_{1(i)}, \ldots, \boldsymbol{h}_{d(i)}\right]$ is an orthogonal matrix of the corresponding eigenvectors. We assume $\lambda_{2(i)}>0$ for $i=1,2$, and $\lambda_{1(i)}$ s are of multiplicity one in the sense that

$$
\liminf _{d \rightarrow \infty} \lambda_{1(i)} / \lambda_{2(i)}>1 \text { for } i=1,2 .
$$

Let $\boldsymbol{X}_{i}-\left[\boldsymbol{\mu}_{i}, \ldots, \boldsymbol{\mu}_{i}\right]=\boldsymbol{H}_{i} \boldsymbol{\Lambda}_{i}^{1 / 2} \boldsymbol{Z}_{i}$ for $i=1,2$. Then, $\boldsymbol{Z}_{i}$ is a $d \times n_{i}$ sphered data matrix from a distribution with the zero mean and identity covariance matrix. Let $\boldsymbol{Z}_{i}=\left[\boldsymbol{z}_{1(i)}, \ldots, \boldsymbol{z}_{d(i)}\right]^{T}$ and $\boldsymbol{z}_{s(i)}=\left(z_{s 1(i)}, \ldots, z_{s n_{i}(i)}\right)^{T}, s=1, \ldots, d$, for $i=1,2$. Note that $E\left(z_{q j(i)} z_{s j(i)}\right)=0(q \neq s)$ and $\operatorname{Var}\left(\boldsymbol{z}_{s(i)}\right)=\boldsymbol{I}_{n_{i}}$, where $\boldsymbol{I}_{n_{i}}$ denotes the $n_{i}$-dimensional identity matrix. Also, note that if $\boldsymbol{X}_{i}$ is Gaussian, $z_{s j(i)}$ s are i.i.d. as the standard normal distribution, $N(0,1)$. We assume that the fourth moments of each variable in $\boldsymbol{Z}_{i}$ are uniformly bounded for $i=1,2$. Also, we assume the following assumption:

(A-i) $\quad E\left(z_{q j(i)}^{2} z_{s j(i)}^{2}\right)=1, E\left(z_{q j(i)} z_{s j(i)} z_{t j(i)}\right)=0$ and $E\left(z_{q j(i)} z_{s j(i)} z_{t j(i)} z_{u j(i)}\right)=$ 0 for all $q \neq s, t, u$.

This kind of assumption was made by Bai and Saranadasa (1996), Chen and Qin (2010) and Aoshima and Yata (2011). We note that (A-i) naturally holds when $\boldsymbol{X}_{i}$ is Gaussian. If $z_{q j(i)} \mathrm{S}$ are independent, (A-i) is met. Another example satisfying (A-i) is the case when $\boldsymbol{X}_{i}$ has a skew normal distribution. See Remark S4.1 in Aoshima and Yata (2018) for the details. When $\boldsymbol{X}_{i}$ has a $d$-dimensional $t$-distribution, the second and third conditions in (A-i) are met while the first condition " $E\left(z_{q j(i)}^{2} z_{s j(i)}^{2}\right)=1$ " is not met. However, as the degree of freedom of the $t$-distribution becomes large, $E\left(z_{q j(i)}^{2} z_{s j(i)}^{2}\right)$ becomes close to 1 for all $q \neq s$. See Section 5 for the details.

We consider the equality test of covariance matrices as follows:

$$
H_{0}: \boldsymbol{\Sigma}_{1}=\boldsymbol{\Sigma}_{2} \quad \text { vs. } \quad H_{1}: \boldsymbol{\Sigma}_{1} \neq \boldsymbol{\Sigma}_{2}
$$

Schott (2007) gave a test procedure based on the Frobenius norm when $d / n_{i} \rightarrow$ $c_{i} \in[0, \infty)$. Srivastava and Yanagihara (2010) considered a test procedure by using a Moore-Penrose inverse covariance matrix. Aoshima and Yata (2011) gave a test procedure based on the quantity of $\operatorname{tr}\left(\Sigma_{1}-\Sigma_{2}\right)$. They also discussed sample size determination so as to have a prespecified size and power simultaneously. Li and Chen (2012) considered the test problem by using the quantity of 
$\operatorname{tr}\left\{\left(\Sigma_{1}-\Sigma_{2}\right)^{2}\right\}$. The above references discussed asymptotic properties of their test procedures when $d \rightarrow \infty$ and $n_{i} \rightarrow \infty$ under the following eigenvalue condition:

$$
\frac{\lambda_{1(i)}^{2}}{\operatorname{tr}\left(\Sigma_{i}^{2}\right)} \rightarrow 0 \text { as } d \rightarrow \infty \text { for } i=1,2 .
$$

Aoshima and Yata (2018) called (1.2) the "non-strongly spiked eigenvalue (NSSE) model". On the other hand, Ishii et al. (2016) investigated asymptotic properties of the first principal component and considered the test problem (1.1) when $d \rightarrow$ $\infty$ while $n_{i}$ s are fixed under the following eigenvalue condition:

$$
\frac{\sum_{s=2}^{d} \lambda_{s(i)}^{2}}{\lambda_{1(i)}^{2}}=o(1) \text { as } d \rightarrow \infty \text { for } i=1,2 .
$$

Note that (1.3) implies the conditions that $\lambda_{2(i)} / \lambda_{1(i)} \rightarrow 0$ and $\lambda_{1(i)}^{2} / \operatorname{tr}\left(\Sigma_{i}^{2}\right) \rightarrow 1$ as $d \rightarrow \infty$. For a spiked model as

$$
\lambda_{s(i)}=a_{s(i)} d^{\alpha_{s(i)}}\left(s=1, \ldots, k_{i}\right) \quad \text { and } \quad \lambda_{s(i)}=c_{s(i)}\left(s=k_{i}+1, \ldots, d\right)
$$

with positive (fixed) constants, $a_{s(i)} \mathrm{s}, c_{s(i)} \mathrm{S}$ and $\alpha_{s(i)} \mathrm{S}$, and a positive (fixed) integer $k_{i}$, the condition (1.3) is met when $\alpha_{1(i)}>1 / 2$ and $\alpha_{1(i)}>\alpha_{2(i)}$. The condition (1.3) is generalized as

(A-ii) $\quad \liminf _{d \rightarrow \infty}\left\{\frac{\lambda_{1(i)}^{2}}{\operatorname{tr}\left(\Sigma_{i}^{2}\right)}\right\}>0$ for $i=1$ and 2 .

For the spiked model (1.4), (A-ii) is met when $\alpha_{1(i)} \geq 1 / 2$ for $i=1,2$. Aoshima and Yata (2018) called (A-ii) the "strongly spiked eigenvalue (SSE) model" and showed that high-dimensional data often have the SSE model. They also provided a method to distinguish between the SSE model and the NSSE model. See Section 5 in Aoshima and Yata (2018). Ishii (2017a, b) considered two-sample tests under (1.3) when $d \rightarrow \infty$ while $n_{i}$ s are fixed. The SSE model (A-ii) is quite difficult to handle because of the influence of strongly spiked noise. In order to handle huge noise, Aoshima and Yata (2018) created a data-transformation technique for twosample tests which transforms the SSE model to the NSSE model. In this paper, we give a new test procedure for (1.1) under the SSE model (A-ii) by using a new approach which is different from the data-transformation technique.

The rest of this paper is organized as follows. In Section 2, we investigate the performance of the test procedure by Li and Chen (2012) under a SSE model. We 
emphasize that one should construct a test procedure by considering eigenstructures of high-dimensional data. We give a modification of their test statistic under (1.3). In Section 3, we consider a test statistic under the SSE model (A-ii). In Section 4, we propose a new test procedure by using the test statistic. We show that the proposed test procedure has consistency properties both for the size and power. In Section 5, we study the performance of the test procedure in numerical simulations. Finally, in Section 6, we give actual data analyses using a microarray data set.

\section{Performance of the earlier test statistic under the SSE model}

In this section, we investigate the performance of the test statistic given by $\mathrm{Li}$ and Chen (2012).

\subsection{The earlier test procedure for (1.1)}

For (1.1), Li and Chen (2012) assumed that

$$
\operatorname{tr}\left(\Sigma_{i} \Sigma_{j} \Sigma_{k} \Sigma_{l}\right)=o\left\{\operatorname{tr}\left(\Sigma_{i} \Sigma_{j}\right) \operatorname{tr}\left(\Sigma_{k} \Sigma_{l}\right)\right\}
$$

for any $i, j, k$ and $l \in\{1,2\}$. Note that (2.1) is one of the NSSE models. They proposed a test statistic as follows:

$$
U_{n_{1}, n_{2}}=A_{n_{1}}+A_{n_{2}}-2 \operatorname{tr}\left(\boldsymbol{S}_{1 n_{1}} \boldsymbol{S}_{2 n_{2}}\right)
$$

where $\boldsymbol{S}_{i n_{i}}$ is the sample covariance matrix having $E\left(\boldsymbol{S}_{i n_{i}}\right)=\boldsymbol{\Sigma}_{i}$ and

$$
\begin{aligned}
A_{n_{i}}= & \frac{1}{n_{i}\left(n_{i}-1\right)} \sum_{j \neq k}^{n_{i}}\left(\boldsymbol{x}_{i j}^{T} \boldsymbol{x}_{i k}\right)^{2}-\frac{2}{n_{i}\left(n_{i}-1\right)\left(n_{i}-2\right)} \sum_{j \neq k \neq l}^{n_{i}} \boldsymbol{x}_{i k}^{T} \boldsymbol{x}_{i j} \boldsymbol{x}_{i j}^{T} \boldsymbol{x}_{i l} \\
& +\frac{1}{n_{i}\left(n_{i}-1\right)\left(n_{i}-2\right)\left(n_{i}-3\right)} \sum_{j \neq k \neq l \neq l^{\prime}}^{n_{i}} \boldsymbol{x}_{i j}^{T} \boldsymbol{x}_{i k} \boldsymbol{x}_{i l}^{T} \boldsymbol{x}_{i l^{\prime}} .
\end{aligned}
$$

Note that $A_{n_{i}}$ is an unbiased estimator of $\operatorname{tr}\left(\Sigma_{i}^{2}\right)$. Thus $U_{n_{1}, n_{2}}$ is an unbiased estimator of $\left\|\Sigma_{1}-\Sigma_{2}\right\|_{F}^{2}=\operatorname{tr}\left\{\left(\Sigma_{1}-\Sigma_{2}\right)^{2}\right\}(=\Delta$, say $)$. Let

$$
m=\min \left\{d, n_{\min }\right\} \text {, where } n_{\min }=\min \left\{n_{1}, n_{2}\right\} .
$$

In this paper, we consider the divergence condition as

$$
d \rightarrow \infty, n_{1} \rightarrow \infty \text { and } n_{2} \rightarrow \infty
$$


which is equivalent to $m \rightarrow \infty$. Note that

$$
\begin{aligned}
\operatorname{Var}\left\{\frac{A_{n_{i}}}{\operatorname{tr}\left(\Sigma_{i}^{2}\right)}\right\} & =\frac{4}{n_{i}^{2}}\{1+o(1)\}+O\left(\frac{\operatorname{tr}\left(\Sigma_{i}^{4}\right)}{\operatorname{tr}\left(\Sigma_{i}^{2}\right)^{2} n_{i}}\right) \rightarrow 0 \\
\text { and } \operatorname{Var}\left(U_{n_{1}, n_{2}}\right)= & \sum_{i=1}^{2}\left(\frac{4 \operatorname{tr}\left(\Sigma_{i}^{2}\right)^{2}}{n_{i}^{2}}\{1+o(1)\}+O\left(\frac{\operatorname{tr}\left\{\left(\Sigma_{i}\left(\Sigma_{1}-\Sigma_{2}\right)\right)^{2}\right\}}{n_{i}}\right)\right) \\
& +\frac{8 \operatorname{tr}\left\{\left(\Sigma_{1} \Sigma_{2}\right)^{2}\right\}}{n_{1} n_{2}}
\end{aligned}
$$

as $m \rightarrow \infty$ under (A-i), so that

$$
\operatorname{Var}\left(U_{n_{1}, n_{2}}\right)=\left(2 \operatorname{tr}\left(\Sigma_{1}^{2}\right) / n_{1}+2 \operatorname{tr}\left(\Sigma_{2}^{2}\right) / n_{2}\right)^{2}\{1+o(1)\} \text { under } H_{0} .
$$

See Sections 2 and 6 in Li and Chen (2012) for the details. Let

$$
T_{\mathrm{LC}}=\frac{U_{n_{1}, n_{2}}}{2 A_{n_{1}} / n_{2}+2 A_{n_{2}} / n_{1}} .
$$

They showed that

$$
T_{\mathrm{LC}} \Rightarrow N(0,1) \text { as } m \rightarrow \infty
$$

under $H_{0},(2.1)$ and some regularity conditions. Here, " $\Rightarrow$ " denotes the convergence in distribution and $N(0,1)$ denotes a random variable distributed as the standard normal distribution.

Let us see the behavior of $T_{\mathrm{LC}}$ under a NSSE model and a SSE model by using a toy example. We set $d=2048$ and $n_{1}=n_{2}=100$. We assumed $N_{d}(\mathbf{0}, \boldsymbol{\Sigma})$ for each class under $H_{0}: \Sigma_{1}=\Sigma_{2}=\Sigma$. We set

$$
\boldsymbol{\Sigma}=\left(\begin{array}{cc}
\boldsymbol{\Sigma}_{(1)} & \boldsymbol{O} \\
\boldsymbol{O} & \boldsymbol{\Sigma}_{(2)}
\end{array}\right) \text { having } \boldsymbol{\Sigma}_{(2)}=\left(0.3^{|i-j|}\right)
$$

and considered two cases:

$$
\text { (i) } \boldsymbol{\Sigma}_{(1)}=\operatorname{diag}\left(d^{1 / 3}, d^{1 / 6}\right) \text { and (ii) } \boldsymbol{\Sigma}_{(1)}=\operatorname{diag}\left(d^{1}, d^{1 / 2}\right) \text {. }
$$

Note that (i) is a NSSE model and (ii) is a SSE model. We generated independent pseudo-random observations from each class and calculated $T_{\mathrm{LC}} 1000$ times. In Fig. 1, we gave histograms for (i) and (ii). One can observe that $T_{\mathrm{LC}}$ converges to $N(0,1)$ in (i), however does not so in (ii). In order to overcome this inconvenience, we first modify $T_{\mathrm{LC}}$ under (1.3) in Section 2.2 and newly construct a different test procedure for the SSE model (A-ii) in Section 4. 


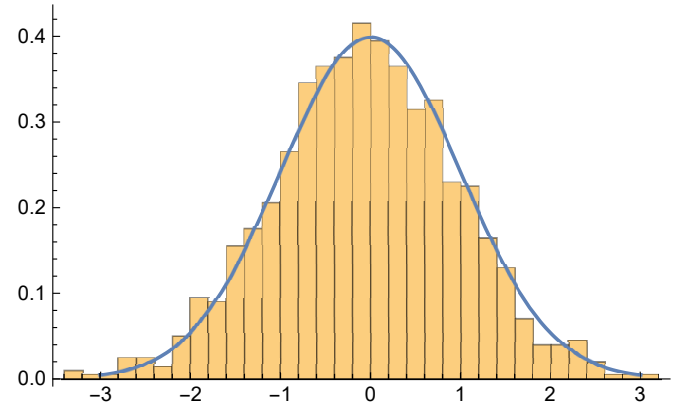

(i) When $\boldsymbol{\Sigma}_{(1)}=\operatorname{diag}\left(d^{1 / 3}, d^{1 / 6}\right)$

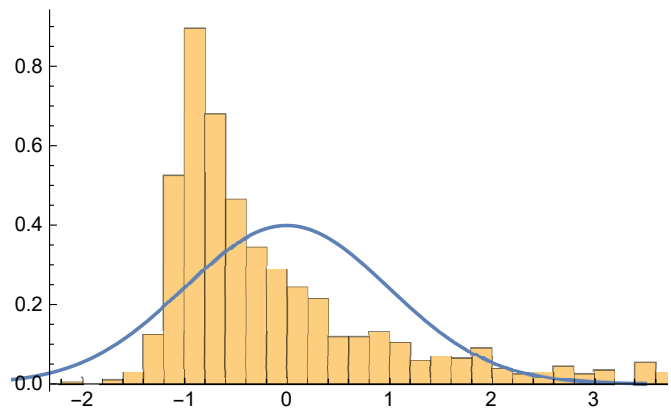

(ii) When $\boldsymbol{\Sigma}_{(1)}=\operatorname{diag}\left(d^{1}, d^{1 / 2}\right)$

Figure 1. The histograms of $T_{\mathrm{LC}}$ for a NSSE model (in the left panel) and for a SSE model (in the right panel). The solid line denotes the p.d.f. of $N(0,1)$.

\subsection{Modification of $T_{L C}$ under a SSE model}

We assume (1.3) as a SSE model. Let

$$
K=2 \lambda_{1(1)}^{2} / n_{1}+2 \lambda_{1(2)}^{2} / n_{2} .
$$

From (2.3), we note that $\operatorname{Var}\left(U_{n_{1}, n_{2}}\right)=K^{2}\{1+o(1)\}$ as $m \rightarrow \infty$ under (Ai), (1.3) and $H_{0}$. We assume the following assumption for the first (normalized) principal component (PC) scores:

(A-iii) $\quad z_{1 j(i)}, j=1, \ldots, n_{i}$, are i.i.d. as $N(0,1)$ for $i=1,2$.

We note that (A-iii) is a Gaussian assumption only for the first PC scores. Thus, (A-iii) is much milder than the Gaussian assumption for $\boldsymbol{X}_{i}$ because $z_{s j(i)}, j=$ $1, \ldots, n_{i} ; s=1, \ldots, d$, are i.i.d. as $N(0,1)$ when $\boldsymbol{X}_{i}$ is Gaussian. Note that $E\left\{\left(z_{1 j(i)}^{2}-1\right)^{2}\right\}=2$ for all $i, j$, under (A-iii). Let

$$
W=\sum_{j=1}^{n_{1}} \frac{\lambda_{1(1)}\left(z_{1 j(1)}^{2}-1\right)}{n_{1}}-\sum_{k=1}^{n_{2}} \frac{\lambda_{1(2)}\left(z_{1 k(2)}^{2}-1\right)}{n_{2}} .
$$

Then, we have that $\operatorname{Var}(W)=E\left(W^{2}\right)=K$. We have the following result.

Lemma 2.1. Under (A-i), (A-iii) and (1.3), it holds that as $m \rightarrow \infty$

$$
\begin{aligned}
U_{n_{1}, n_{2}}= & \left(W+\lambda_{1(1)}-\lambda_{1(2)}\right)^{2}+\Delta-\left(\lambda_{1(1)}-\lambda_{1(2)}\right)^{2}-K+o_{p}(K) \\
& +O_{p}\left[n_{\max }^{1 / 2}\left\{1-\left(\boldsymbol{h}_{1(1)}^{T} \boldsymbol{h}_{1(2)}\right)^{2}\right\} K\right]+o_{p}\left\{(K \Delta)^{1 / 2}\right\},
\end{aligned}
$$

where $n_{\max }=\max \left\{n_{1}, n_{2}\right\}$. 
From Lemma 2.1, under (A-i), (A-iii), (1.3) and $H_{0}$, it holds that as $m \rightarrow \infty$

$$
U_{n_{1}, n_{2}}=W^{2}-K+o_{p}(K) .
$$

Let $T_{n_{1}, n_{2}}=U_{n_{1}, n_{2}} / K+1$. Then, we have an asymptotic distribution of $T_{n_{1}, n_{2}}$ under $H_{0}$.

Proposition 2.1. Under (A-i), (A-iii), (1.3) and $H_{0}$, it holds that $T_{n_{1}, n_{2}} \Rightarrow \chi_{1}^{2}$ as $m \rightarrow \infty$. Here, $\chi_{\nu}^{2}$ denotes a random variable distributed as a $\chi^{2}$ distribution with $\nu$ degrees of freedom.

Since $\lambda_{1(i)} \mathrm{S}$ are unknown, we need to estimate them. It is well known that the sample eigenvalues involve too much noise for high-dimensional data. See Jung and Marron (2009), Ishii et al. (2016) and Shen et al. (2016) for the details. Also, see Appendix A.1. We consider estimating $\lambda_{1(i)}$ s by using the noise-reduction (NR) methodology given by Yata and Aoshima $(2012,2013)$. Let $\overline{\boldsymbol{X}}_{i}=\left[\overline{\boldsymbol{x}}_{i}, \ldots, \overline{\boldsymbol{x}}_{i}\right]$ and $\overline{\boldsymbol{x}}_{i}=n_{i}^{-1} \sum_{j=1}^{n_{i}} \boldsymbol{x}_{i j}$ for $i=1,2$. We denote the dual matrix of $\boldsymbol{S}_{i n_{i}}$ by $\boldsymbol{S}_{i D}$ and define its eigen-decomposition as follows:

$$
\boldsymbol{S}_{i D}=\left(n_{i}-1\right)^{-1}\left(\boldsymbol{X}_{i}-\overline{\boldsymbol{X}}_{i}\right)^{T}\left(\boldsymbol{X}_{i}-\overline{\boldsymbol{X}}_{i}\right)=\sum_{s=1}^{n_{i}-1} \hat{\lambda}_{s(i)} \hat{\boldsymbol{u}}_{s(i)} \hat{\boldsymbol{u}}_{s(i)}^{T},
$$

where $\hat{\lambda}_{1(i)} \geq \cdots \geq \hat{\lambda}_{n_{i}-1(i)}(\geq 0)$ and $\hat{\boldsymbol{u}}_{s(i)}$ denotes a unit eigenvector corresponding to the eigenvalue $\hat{\lambda}_{s(i)}$. Note that $\boldsymbol{S}_{i n_{i}}$ and $\boldsymbol{S}_{i D}$ share non-zero eigenvalues. If one uses the NR method, $\lambda_{j(i)} \mathrm{S}$ are estimated by

$$
\tilde{\lambda}_{j(i)}=\hat{\lambda}_{j(i)}-\frac{\operatorname{tr}\left(\boldsymbol{S}_{i D}\right)-\sum_{s=1}^{j} \hat{\lambda}_{s(i)}}{n_{i}-1-j} \quad\left(j=1, \ldots, n_{i}-2\right) .
$$

Note that $\tilde{\lambda}_{j(i)} \geq 0$ w.p. 1 for $j=1, \ldots, n_{i}-2$. See Appendix A.1 for asymptotic properties of $\tilde{\lambda}_{1(i)}$ s. Let

$$
\widetilde{T}_{n_{1}, n_{2}}=U_{n_{1}, n_{2}} / \widetilde{K}+1 \text { with } \widetilde{K}=2 \tilde{\lambda}_{1(1)}^{2} / n_{1}+2 \tilde{\lambda}_{1(2)}^{2} / n_{2}
$$

Then, we have the following result.

Theorem 2.1. Under (A-i), (A-iii), (1.3) and $H_{0}$, it holds that as $m \rightarrow \infty$

$$
\widetilde{T}_{n_{1}, n_{2}} \Rightarrow \chi_{1}^{2}
$$


We consider testing (1.1) for a given $\alpha \in(0,1 / 2)$ by

$$
\text { rejecting } H_{0} \Longleftrightarrow \widetilde{T}_{n_{1}, n_{2}} \geq c_{1}(\alpha),
$$

where $c_{1}(\alpha)$ denotes the upper $\alpha$ point of $\chi_{1}^{2}$. Then, under (A-i), (A-iii) and (1.3), it holds that as $m \rightarrow \infty$

$$
\text { size }=\alpha+o(1)
$$

Remark 2.1. We note that $A_{n_{i}}^{1 / 2}$ is a consistent estimator of $\lambda_{1(i)}$ under (1.3) from the fact that $A_{n_{i}} / \operatorname{tr}\left(\Sigma_{i}^{2}\right)=A_{n_{i}} /\left[\lambda_{1(i)}^{2}\{1+o(1)\}\right]=1+o_{p}(1)$ as $m \rightarrow \infty$ under (A-i) and (1.3). Then, it holds that $T_{\mathrm{LC}}+1=\widetilde{T}_{n_{1}, n_{2}}+o_{p}(1)$ under (A-i), (1.3) and $H_{0}$ because $\widetilde{K} /\left(2 A_{n_{1}} / n_{2}+2 A_{n_{2}} / n_{1}\right)=1+o_{p}(1)$. Thus, one may use $T_{\mathrm{LC}}+1$ for $\widetilde{T}_{n_{1}, n_{2}}$ in (2.6). However, we recommend using $\tilde{\lambda}_{1(i)}$ for estimation of $\lambda_{1(i)}$ because $\tilde{\lambda}_{1(i)}$ has a consistency property and the asymptotic normality even when the condition (1.3) is not met. On the other hand, $\hat{\lambda}_{1(i)}$ does not have the consistency property even when (1.3) is met. See Appendix A.1 for the details.

Next, we consider the power of $\widetilde{T}_{n_{1}, n_{2}}$. Throughout this paper, we denote the power of a test statistic, $T$ (say), by $\operatorname{Power}(T)$. We note that $K$ is the main term in the standard deviation of $U_{n_{1}, n_{2}}$. By noting that

$$
\begin{aligned}
& \operatorname{tr}\left(\boldsymbol{\Sigma}_{1} \boldsymbol{\Sigma}_{2}\right) /\left(n_{1} n_{2}\right) \leq\left\{\operatorname{tr}\left(\boldsymbol{\Sigma}_{1}^{2}\right) \operatorname{tr}\left(\boldsymbol{\Sigma}_{2}^{2}\right) /\left(n_{1} n_{2}\right)^{2}\right\}^{1 / 2}=O\left(K^{2}\right) \\
& \text { and } \operatorname{tr}\left[\left\{\boldsymbol{\Sigma}_{i}\left(\boldsymbol{\Sigma}_{1}-\boldsymbol{\Sigma}_{2}\right)\right\}^{2}\right] / n_{i} \leq \lambda_{1(i)}^{2} \Delta / n_{i}=O(K \Delta),
\end{aligned}
$$

from (2.3), it holds that $\operatorname{Var}\left(U_{n_{1}, n_{2}}\right) / \Delta^{2}=o(1)$ as $m \rightarrow \infty$ under (A-i) and $K / \Delta=o(1)$ as $m \rightarrow \infty$, so that

$$
U_{n_{1}, n_{2}} / \Delta=1+o_{p}(1) .
$$

Here, " $K / \Delta=o(1)$ " means that the intrinsic information about $\Sigma_{1}-\Sigma_{2}$ is larger than the noise. Thus, if the difference between $\Sigma_{i} \mathrm{~s}$ is quite large enough to claim $K / \Delta=o(1)$ as $m \rightarrow \infty, U_{n_{1}, n_{2}}$ has the consistency. Let $\Delta_{1}=$ $\Delta-\left(\lambda_{1(1)}-\lambda_{1(2)}\right)^{2}$ and $\omega=\left(\lambda_{1(1)}-\lambda_{1(2)}\right) / K^{1 / 2}$. From Lemma 2.1 and (B.9) in Appendix B of the online supplementary, it holds that $W / K^{1 / 2} \Rightarrow N(0,1)$ and

$$
\left(U_{n_{1}, n_{2}}-\Delta_{1}\right) / K+1=\left(W / K^{1 / 2}+\omega\right)^{2}+o_{p}(1) \Rightarrow \chi_{1}^{2}\left(\omega^{2}\right) \text { as } m \rightarrow \infty
$$

under (A-i), (A-iii), (1.3) and the conditions:

$$
\left|\boldsymbol{h}_{1(1)}^{T} \boldsymbol{h}_{1(2)}\right|=1+o\left(n_{\max }^{-1 / 2}\right) \text { as } m \rightarrow \infty \text { and } \limsup _{m \rightarrow \infty} \frac{\Delta}{K}<\infty .
$$


Here, $\chi_{1}^{2}\left(\omega^{2}\right)$ denotes a random variable distributed as a noncentral $\chi^{2}$ distribution having the noncentrality parameter $\omega^{2}$ with 1 degree of freedom. An intuitive meaning of the second condition in (2.8) is that the noise is as large as the intrinsic information or more. Thus, if the difference between $\Sigma_{i}$ s is not large enough to claim $K / \Delta=o(1)$ as $m \rightarrow \infty, U_{n_{1}, n_{2}}$ has the asymptotic distribution. Then, we have the following results.

Theorem 2.2. Assume (A-i) and (1.3). Under $K / \Delta=o(1)$ as $m \rightarrow \infty$, it holds that as $m \rightarrow \infty$

$$
\text { Power }\left(\widetilde{T}_{n_{1}, n_{2}}\right)=1+o(1) \text {. }
$$

Under (A-iii) and (2.8), it holds that as $m \rightarrow \infty$

$$
\operatorname{Power}\left(\widetilde{T}_{n_{1}, n_{2}}\right)=1-F_{\omega^{2}}\left(c_{1}(\alpha)-\Delta_{1} / K\right)+o(1),
$$

where $F_{\omega^{2}}(\cdot)$ denotes the c.d.f. of $\chi_{1}^{2}\left(\omega^{2}\right)$.

If the difference between $\Sigma_{i}$ s is quite large enough to claim $K / \Delta=o(1)$ as $m \rightarrow \infty$, the power tends to 1 . For example, we have the following result.

Corollary 2.1. Assume (A-i) and (1.3). Under either $\lim _{\sup } \operatorname{si\infty }\left|\boldsymbol{h}_{1(1)}^{T} \boldsymbol{h}_{1(2)}\right|<1$ or $\liminf _{d \rightarrow \infty}\left|\lambda_{1(1)} / \lambda_{1(2)}-1\right|>0$, we have (2.9) as $m \rightarrow \infty$.

Now, we consider the following alternative hypothesis:

$$
H_{1 a}:\left(\lambda_{1(1)}, \boldsymbol{h}_{1(1)}\right)=\left(\lambda_{1(2)}, \boldsymbol{h}_{1(2)}\right) \text { and } \boldsymbol{\Sigma}_{1 \star} \neq \boldsymbol{\Sigma}_{2 \star},
$$

where $\boldsymbol{\Sigma}_{i \star}=\sum_{s=2}^{d} \lambda_{s(i)} \boldsymbol{h}_{s(i)} \boldsymbol{h}_{s(i)}^{T}$ for $i=1,2$. We assume a situation that

$$
\left\|\Sigma_{1 \star}-\Sigma_{2 \star}\right\|_{F}^{2} / K=o(1) \text { as } m \rightarrow \infty \text {. }
$$

In this situation, we have that $\Delta=\Delta_{1}=\left\|\Sigma_{1 \star}-\Sigma_{2 \star}\right\|_{F}^{2}$. From (2.10) it holds that as $m \rightarrow \infty$

$$
\operatorname{Power}\left(\widetilde{T}_{n_{1}, n_{2}}\right)=1-F_{0}\left(c_{1}(\alpha)\right)+o(1)=\alpha+o(1)
$$

under (A-i), (A-iii) and (1.3).

Remark 2.2. The condition (2.11) is met when $\operatorname{tr}\left(\Sigma_{i \star}^{2}\right) / \lambda_{1(i)}^{2}=o\left(n_{i}^{-1}\right)$ for $i=$ 1,2. It often occurs under the SSE model (A-ii).

From (2.12) and Remark 2.2, one should note that $\widetilde{T}_{n_{1}, n_{2}}$ cannot distinguish the two covariance matrices when they share the first eigenspace. It is because under (1.3) the strongly-spiked first eigenvalue obscures the difference between $\Sigma_{1 \star}$ and $\Sigma_{2 \star}$. Although (1.3) is one of the SSE models, it is limited in actual data analyses. In Section 3, we newly consider a test procedure in general under the SSE model (A-ii). 


\section{A test statistic for the SSE model}

In this section, we consider a test statistic under the SSE model (A-ii). From the fact that $\operatorname{tr}\left(\Sigma_{1 \star} \Sigma_{2 \star}\right) \leq\left\{\operatorname{tr}\left(\Sigma_{1 \star}^{2}\right) \operatorname{tr}\left(\Sigma_{2 \star}^{2}\right)\right\}^{1 / 2} \leq \operatorname{tr}\left(\Sigma_{1 \star}^{2}\right)+\operatorname{tr}\left(\Sigma_{2 \star}^{2}\right)$, we note that $\boldsymbol{h}_{1(i)}^{T} \boldsymbol{\Sigma}_{i^{\prime} \star} \boldsymbol{h}_{1(i)}=o\left(\lambda_{1\left(i^{\prime}\right)}\right)$ for $i \neq i^{\prime}$ and $\left\|\boldsymbol{\Sigma}_{1 \star}-\boldsymbol{\Sigma}_{2 \star}\right\|_{F}^{2}=o\left(\lambda_{1(1)}^{2}+\lambda_{1(2)}^{2}\right)$ as $d \rightarrow \infty$ under (1.3). Then, it holds that as $d \rightarrow \infty$

$$
\Delta=\left(\lambda_{1(1)}-\lambda_{1(2)}\right)^{2}+2 \lambda_{1(1)} \lambda_{1(2)}\left\{1-\left(\boldsymbol{h}_{1(1)}^{T} \boldsymbol{h}_{1(2)}\right)^{2}\right\}+o\left(\lambda_{1(1)}^{2}+\lambda_{1(2)}^{2}\right) .
$$

We consider (3.1) as a starting point to handle the SSE model (A-ii). In this section, we give a test statistic based on (3.1) and show that it holds an asymptotic null distribution even when (1.3) is not met. In Section 4, we improve the test statistic to detect the disparity between $\Sigma_{1 \star}$ and $\Sigma_{2 \star}$ and propose a new test procedure for the SSE model (A-ii). By using the NR method, we estimate the first eigenvector as

$$
\tilde{\boldsymbol{h}}_{1(i)}=\left\{\left(n_{i}-1\right) \tilde{\lambda}_{1(i)}\right\}^{-1 / 2}\left(\boldsymbol{X}_{i}-\overline{\boldsymbol{X}}_{i}\right) \hat{\boldsymbol{u}}_{1(i)}
$$

for $i=1,2$, where $\hat{\boldsymbol{u}}_{1(i)}$ is given in (2.4). Note that $\tilde{\boldsymbol{h}}_{1(i)}=\left(\hat{\lambda}_{1(i)} / \tilde{\lambda}_{1(i)}\right)^{1 / 2} \hat{\boldsymbol{h}}_{1(i)}$, where $\hat{\boldsymbol{h}}_{1(i)}$ is the first (unit) eigenvector of $\boldsymbol{S}_{i n_{i}}$. Let $\delta_{i}=\operatorname{tr}\left(\boldsymbol{\Sigma}_{i \star}^{2}\right), i=1,2$. From Lemma A.1 in Appendix A.1, it holds that as $m \rightarrow \infty$

$$
\left|\tilde{\boldsymbol{h}}_{1(1)}^{T} \tilde{\boldsymbol{h}}_{1(2)}\right|=1+O_{p}\left(\frac{\delta_{1}^{1 / 2}}{n_{1} \lambda_{1(1)}}+\frac{\delta_{2}^{1 / 2}}{n_{2} \lambda_{1(2)}}\right)=1+o_{p}\left(n_{\min }^{-1}\right)
$$

under (A-i), (1.3) and $H_{0}$, where $n_{\min }$ and $m$ are given in (2.2). Then, from (3.1) we consider the following quantity:

$$
V=\frac{\left(\tilde{\lambda}_{1(1)}-\tilde{\lambda}_{1(2)}\right)^{2}+2 \tilde{\lambda}_{1(1)} \tilde{\lambda}_{1(2)}\left\{1-\min \left\{1,\left(\tilde{\boldsymbol{h}}_{1(1)}^{T} \tilde{\boldsymbol{h}}_{1(2)}\right)^{2}\right\}\right\}}{\sum_{i=1}^{2} 2 \tilde{\lambda}_{1(i)}^{2} /\left(n_{i}-1\right)} .
$$

From Lemma A.1 and (3.2), it holds that as $m \rightarrow \infty$

$$
\begin{aligned}
& \frac{\tilde{\lambda}_{1(1)}-\tilde{\lambda}_{1(2)}}{\left\{\sum_{i=1}^{2} 2 \lambda_{1(i)}^{2} /\left(n_{i}-1\right)\right\}^{1 / 2}}=\frac{W}{K^{1 / 2}}+o_{p}(1) \Rightarrow N(0,1), \\
& \text { and } \frac{\tilde{\lambda}_{1(1)} \tilde{\lambda}_{1(2)}\left\{1-\min \left\{1,\left(\tilde{\boldsymbol{h}}_{1(1)}^{T} \tilde{\boldsymbol{h}}_{1(2)}\right)^{2}\right\}\right\}}{\left\{\sum_{i=1}^{2} 2 \lambda_{1(i)}^{2} /\left(n_{i}-1\right)\right\}}=o_{p}(1)
\end{aligned}
$$

under (A-i), (A-iii), (1.3) and $H_{0}$. Then, we have the following result. 
Proposition 3.1. Under (A-i), (A-iii), (1.3) and $H_{0}$, it holds that $V \Rightarrow \chi_{1}^{2}$ as $m \rightarrow \infty$.

From (3.3) it holds that $V=W^{2} / K+o_{p}(1)$ as $m \rightarrow \infty$ under (A-i), (A-iii), (1.3) and $H_{0}$. Thus, from Lemmas 2.1 and A.1, $V$ is asymptotically equivalent to $\widetilde{T}_{n_{1}, n_{2}}$ under (1.3) and $H_{0}$. However, as for $V$, one can consider it in general under the SSE model (A-ii) as follows: If $\lim _{\sup _{d \rightarrow \infty}} \lambda_{1(i)} / \lambda_{2(i)}<\infty$ for some $i$, it holds from Lemma A.1 that

$$
1-\min \left\{1,\left(\tilde{\boldsymbol{h}}_{1(1)}^{T} \tilde{\boldsymbol{h}}_{1(2)}\right)^{2}\right\}=O_{p}\left(n_{\min }^{-1}\right)
$$

under $H_{0}$, (A-i) and (A-ii), so that (3.3) does not hold. Thus, one cannot ignore the bias of $1-\min \left\{1,\left(\tilde{\boldsymbol{h}}_{1(1)}^{T} \tilde{\boldsymbol{h}}_{1(2)}\right)^{2}\right\}$ in $V$ especially when $\lambda_{2(i)}$ is close to $\lambda_{1(i)}$. In order to reduce the bias, we consider modifying $V$. Let $\eta=\delta_{1}^{1 / 2} / \lambda_{1(1)}+\delta_{2}^{1 / 2} / \lambda_{1(2)}$. From Lemma A.1, it follows that

$$
\left\{1-\min \left\{1,\left(\tilde{\boldsymbol{h}}_{1(1)}^{T} \tilde{\boldsymbol{h}}_{1(2)}\right)^{2}\right\}\right\}^{1+\eta}=o_{p}\left(n_{\min }^{-1}\right)
$$

under $H_{0}$, (A-i) and (A-ii). By using the cross-data-matrix (CDM) method by Yata and Aoshima (2010), we have a consistent estimator of $\delta_{i}, \hat{\delta}_{i}$. See (A.1) in Appendix A.2 for the details. We estimate $\eta$ by

$$
\hat{\eta}=\hat{\delta}_{1}^{1 / 2} / \tilde{\lambda}_{1(1)}+\hat{\delta}_{2}^{1 / 2} / \tilde{\lambda}_{1(2)}
$$

We provide the following test statistic:

$$
T_{\mathrm{NR}}=\frac{\left(\tilde{\lambda}_{1(1)}-\tilde{\lambda}_{1(2)}\right)^{2}+2 \tilde{\lambda}_{1(1)} \tilde{\lambda}_{1(2)}\left\{1-\min \left\{1,\left(\tilde{\boldsymbol{h}}_{1(1)}^{T} \tilde{\boldsymbol{h}}_{1(2)}\right)^{2}\right\}\right\}^{1+\hat{\eta}}}{\sum_{i=1}^{2} 2 \tilde{\lambda}_{1(i)}^{2} /\left(n_{i}-1\right)} .
$$

Then, we have the following result.

Proposition 3.2. Under (A-i) to (A-iii) and $H_{0}$, it holds that $T_{N R} \Rightarrow \chi_{1}^{2}$ as $m \rightarrow \infty$.

Note that one can use $T_{\mathrm{NR}}$ even when (1.3) is not met.

\section{New test procedure for the SSE model}

In view of (2.12), one may regard $\widetilde{T}_{n_{1}, n_{2}}$ and $T_{\mathrm{NR}}$ as test statistics for the equality test of the first eigenspaces from the two classes. In order to distinguish the remaining eigenspaces, we investigate the disparity between $\Sigma_{1 \star}$ and $\Sigma_{2 \star}$. We 
take into account the quantities, $\operatorname{tr}\left(\Sigma_{i \star}\right) \mathrm{s}$ and $\operatorname{tr}\left(\Sigma_{i \star}^{2}\right) \mathrm{s}$, for $T_{\mathrm{NR}}$. Srivastava and Yanagihara (2010) also considered a test statistic for (1.1) by using those quantities. Let $\kappa_{i}=\operatorname{tr}\left(\Sigma_{i \star}\right)$ for $i=1,2$. By noting that $\kappa_{1} / \kappa_{2}=1$ under $H_{0}$, one may consider a test statistic such as $\left(\kappa_{1} / \kappa_{2}\right) T_{\mathrm{NR}}$ or $\left(\kappa_{1} / \kappa_{2}\right)^{-1} T_{\mathrm{NR}}$. We first consider a modification of $T_{\mathrm{NR}}$ by

$$
\kappa_{\star} T_{\mathrm{NR}} \text { with } \kappa_{\star}=\left(\kappa_{1} / \kappa_{2}+\kappa_{2} / \kappa_{1}\right) / 2 .
$$

Note that $\kappa_{\star} \geq 1$ and $\kappa_{\star}=1$ under $H_{0}$. By using the NR method, Ishii et al. (2016) gave an estimator of $\kappa_{i}$ as $\hat{\kappa}_{i}=\operatorname{tr}\left(\boldsymbol{S}_{i D}\right)-\tilde{\lambda}_{1(i)}$, where $\tilde{\lambda}_{1(i)}$ is given by (2.5). We estimate $\kappa_{\star}$ by

$$
\tilde{\kappa}_{\star}=\left(\hat{\kappa}_{1} / \hat{\kappa}_{2}+\hat{\kappa}_{2} / \hat{\kappa}_{1}\right) / 2 .
$$

Then, it holds from Lemma A.3 in Appendix A.2 that $\tilde{\kappa}_{\star}=\kappa_{\star}+o_{p}(1)$ as $m \rightarrow \infty$ under (A-i) and (A-ii). Similarly, we consider $\delta_{i}=\operatorname{tr}\left(\Sigma_{i \star}^{2}\right)$. Let $\delta_{\star}=\left(\delta_{1} / \delta_{2}+\right.$ $\left.\delta_{2} / \delta_{1}\right) / 2$. Note that $\delta_{\star} \geq 1$ and $\delta_{\star}=1$ under $H_{0}$. We estimate $\delta_{\star}$ by

$$
\tilde{\delta}_{\star}=\left(\hat{\delta}_{1} / \hat{\delta}_{2}+\hat{\delta}_{2} / \hat{\delta}_{1}\right) / 2 \text {, }
$$

where $\hat{\delta}_{i}$ s are given by (A.1) in Appendix A.2. Then, it holds from Lemma A.2 that $\tilde{\delta}_{\star}=\delta_{\star}+o_{p}(1)$ as $m \rightarrow \infty$ under (A-i) and (A-ii). Finally, we propose the following test statistic:

$$
T_{\mathrm{IYA}}=\tilde{\kappa}_{\star} \tilde{\delta}_{\star} T_{\mathrm{NR}} .
$$

Remark 4.1. The test statistic $T_{\mathrm{IYA}}$ introduces the differences of $\kappa_{i} \mathrm{~s}$ and $\delta_{i} \mathrm{~s}$ into the definition to improve on the power of $T_{\mathrm{NR}}$. If $\Sigma_{1 \star}=\Sigma_{2 \star}, T_{\mathrm{IYA}}$ becomes close to $T_{\mathrm{NR}}$.

Theorem 4.1. Under (A-i) to (A-iii) and $H_{0}$, it holds that as $m \rightarrow \infty$

$$
T_{I Y A} \Rightarrow \chi_{1}^{2} \text {. }
$$

From Theorem 4.1 we consider testing (1.1) by (2.6) with $T_{\mathrm{IYA}}$ instead of $\widetilde{T}_{n_{1}, n_{2}}$. Then, the size becomes close to $\alpha$ as $m \rightarrow \infty$. Let

$$
\Delta_{\star}=\left(\lambda_{1(1)}-\lambda_{1(2)}\right)^{2}+2 \lambda_{1(1)} \lambda_{1(2)}\left\{1-\left(\boldsymbol{h}_{1(1)}^{T} \boldsymbol{h}_{1(2)}\right)^{2}\right\}^{1+\eta+\gamma},
$$

where $\gamma$ is a positive constant (not depending on $d$ and $n$ ). From Lemmas A.1 and A.2, it follows under (A-i) and (A-ii) that as $m \rightarrow \infty$

$$
\begin{aligned}
\left\{1-\min \left\{1,\left(\tilde{\boldsymbol{h}}_{1(1)}^{T} \tilde{\boldsymbol{h}}_{1(2)}\right)^{2}\right\}\right\}^{1+\hat{\eta}} & =\left\{1-\min \left\{1,\left(\tilde{\boldsymbol{h}}_{1(1)}^{T} \tilde{\boldsymbol{h}}_{1(2)}\right)^{2}\right\}\right\}^{1+\eta+o_{p}(1)} \\
& \geq\left\{1-\min \left\{1,\left(\tilde{\boldsymbol{h}}_{1(1)}^{T} \tilde{\boldsymbol{h}}_{1(2)}\right)^{2}\right\}\right\}^{1+\eta+\gamma}
\end{aligned}
$$

with probability tending to 1 . Then, we have the following result. 
Theorem 4.2. Assume (A-i) and (A-ii). Under $K / \Delta_{\star}=o(1)$ as $m \rightarrow \infty$, it holds that as $m \rightarrow \infty$

$$
\operatorname{Power}\left(T_{I Y A}\right)=1+o(1) .
$$

Under (A-iii), it holds that as $m \rightarrow \infty$

$$
\operatorname{Power}\left(T_{I Y A}\right) \geq 1-F_{\omega^{2}}\left(\kappa_{\star}^{-1} \delta_{\star}^{-1} c_{1}(\alpha)\right)+o(1),
$$

where $F_{\omega^{2}}(\cdot)$ is given in Theorem 2.2.

Corollary 4.1. Assume (A-i) and (A-ii). Under either $\lim _{\sup } \operatorname{sum}_{d \rightarrow \infty}\left|\boldsymbol{h}_{1(1)}^{T} \boldsymbol{h}_{1(2)}\right|<1$ or $\liminf _{d \rightarrow \infty}\left|\lambda_{1(1)} / \lambda_{1(2)}\right|>0$, we have (4.2) as $m \rightarrow \infty$.

If (1.3) is met, we have the following result.

Corollary 4.2. Assume $(A-i)$ and (1.3). Under $\liminf \operatorname{in}_{m \rightarrow \infty} n_{\min }^{\beta}\left(1-\left|\boldsymbol{h}_{1(1)}^{T} \boldsymbol{h}_{1(2)}\right|\right)>$ 0 for some constant $\beta \in(0,1)$, we have (4.2) as $m \rightarrow \infty$.

Let us compare $T_{\text {IYA }}$ with $\widetilde{T}_{n_{1}, n_{2}}$. First, note that $\widetilde{T}_{n_{1}, n_{2}}$ does not hold Theorem 2.1 under the SSE model (A-ii). Since the condition (2.11) is often met under (1.3) as stated in Remark 2.2, we assume (1.3) and (2.11) together. Under (A-i), (1.3), (2.11) and $\boldsymbol{h}_{1(1)}=\boldsymbol{h}_{1(2)}$, it follows from Theorems 2.2 and 4.2 that as $m \rightarrow \infty$

$$
\begin{aligned}
& \operatorname{Power}\left(T_{\mathrm{IYA}}\right) \geq 1-F_{\omega^{2}}\left(\kappa_{\star}^{-1} \delta_{\star}^{-1} c_{1}(\alpha)\right)+o(1), \\
& \operatorname{Power}\left(\widetilde{T}_{n_{1}, n_{2}}\right)=1-F_{\omega^{2}}\left(c_{1}(\alpha)\right)+o(1)
\end{aligned}
$$

from the fact that $\Delta_{1}=\left\|\boldsymbol{\Sigma}_{1 \star}-\boldsymbol{\Sigma}_{2 \star}\right\|_{F}^{2}=o(K)$ under (2.11) and $\boldsymbol{h}_{1(1)}=$ $\boldsymbol{h}_{1(2)}$. Since $F_{\omega^{2}}\left(\kappa_{\star}^{-1} \delta_{\star}^{-1} c_{1}(\alpha)\right) \leq F_{\omega^{2}}\left(c_{1}(\alpha)\right)$, we can claim that $\operatorname{Power}\left(T_{\mathrm{IYA}}\right) \geq$ Power $\left(\widetilde{T}_{n_{1}, n_{2}}\right)$ asymptotically. On the other hand, when $\boldsymbol{h}_{1(1)} \neq \boldsymbol{h}_{1(2)}$, the power of $T_{\mathrm{IYA}}$ often has the consistency property as seen in Corollaries 4.1 and 4.2. Thus, we recommend using $T_{\mathrm{IYA}}$ when the data fits the SSE model (A-ii).

\section{Numerical results and discussions}

In this section, we summarize simulation studies of the findings. We checked the performance of our new test procedure under several types of the SSE model. In addition, we provided a simulation result under a NSSE model in Appendix $\mathrm{C}$ of the online supplementary. We used computer simulations to study performances of the test statistics, $T_{\mathrm{LC}}, \widetilde{T}_{n_{1}, n_{2}}, \tilde{\kappa}_{\star} \tilde{\delta}_{\star} V\left(=T_{V}\right.$, say) and $T_{\mathrm{IYA}}$. Here, we 
examined the influence of $\hat{\eta}$ in $T_{\text {IYA }}$ by $T_{V}$. Remember that $T_{\mathrm{NR}}$ was given by introducing $\hat{\eta}$ into $V$, and $T_{\mathrm{IYA}}=\tilde{\kappa}_{\star} \tilde{\delta}_{\star} T_{\mathrm{NR}}$. We set $\left(n_{1}, n_{2}\right)=\left(3\left\lceil d^{1 / 2}\right\rceil, 4\left\lceil d^{1 / 2}\right\rceil\right)$ and $d=2^{s}$ for $s=7, \ldots, 13$, where $\lceil x\rceil$ denotes the smallest integer $\geq x$. We set $\alpha=0.05$ and

$$
\boldsymbol{\Sigma}_{i}=\left(\begin{array}{cc}
\boldsymbol{\Sigma}_{1(i)} & \boldsymbol{O} \\
\boldsymbol{O} & \boldsymbol{\Sigma}_{2(i)}
\end{array}\right), i=1,2 .
$$

First, we generated independent pseudo-random observations from $N_{d}\left(\mathbf{0}, \boldsymbol{\Sigma}_{i}\right)$ for $\pi_{i}, i=1,2$. We set

$$
\boldsymbol{\Sigma}_{1(1)}=\operatorname{diag}\left(d^{\alpha_{1}}, c_{1} d^{\alpha_{2}}\right) \text { and } \boldsymbol{\Sigma}_{2(1)}=\left(0.2^{|i-j|^{1 / 3}}\right)
$$

for $i=1$ in (5.1). As for the alternative hypothesis, we considered two cases:

(i) $\boldsymbol{\Sigma}_{1(2)}=1.5 \operatorname{diag}\left(d^{\alpha_{1}}, c_{1} d^{\alpha_{2}}\right)$ and $\boldsymbol{\Sigma}_{2(2)}=1.5\left(0.4^{|i-j|^{1 / 3}}\right)$, and

(ii) $\boldsymbol{\Sigma}_{1(2)}=\boldsymbol{B} \operatorname{diag}\left(d^{\alpha_{1}}, c_{1} d^{\alpha_{2}}\right) \boldsymbol{B}$ and $\boldsymbol{\Sigma}_{2(2)}=\left(0.4^{|i-j|^{1 / 3}}\right)$ having

$$
\boldsymbol{B}=\left(\begin{array}{cc}
\cos \theta & \sin \theta \\
\sin \theta & -\cos \theta
\end{array}\right) .
$$

Note that $\lambda_{1(1)} / \lambda_{1(2)}=2 / 3, \boldsymbol{h}_{1(1)}^{T} \boldsymbol{h}_{1(2)}=1, \kappa_{1} \neq \kappa_{2}$ and $\delta_{1} \neq \delta_{2}$ in (i), and $\lambda_{1(1)} / \lambda_{1(2)}=1, \boldsymbol{h}_{1(1)}^{T} \boldsymbol{h}_{1(2)}=\cos \theta, \kappa_{1}=\kappa_{2}$ and $\delta_{1} \neq \delta_{2}$ in (ii). For $\alpha_{1}, \alpha_{2}$ and $c_{1}$, we considered three cases:

(a) $\alpha_{1}=1, \alpha_{2}=1 / 3$ and $c_{1}=1 ; \quad$ (b) $\alpha_{1}=1, \alpha_{2}=1$ and $c_{1}=1 / 3$

and (c) $\alpha_{1}=1 / 2, \alpha_{2}=1 / 3$ and $c_{1}=1$.

Note that (A-ii) holds for (a) to (c), however, (1.3) holds only for (a). For (ii), we set $\theta=\pi / 6$ for (a) and (b), and $\theta=\pi / 4$ for (c) in the matrix $\boldsymbol{B}$.

For each case, we checked the performance by 2000 replications. We defined $P_{r}=1$ (or 0 ) when $H_{0}$ was falsely rejected (or not) for $r=1, \ldots, 2000$, and defined $\bar{\alpha}=\sum_{r=1}^{2000} P_{r} / 2000$ to estimate the size. We also defined $P_{r}=1$ (or 0 ) when $H_{1}$ was falsely rejected (or not) for $r=1, \ldots, 2000$, and defined $1-\bar{\beta}=$ $1-\sum_{r=1}^{2000} P_{r} / 2000$ to estimate the power for (i) and (ii). Note that their standard deviations are less than 0.011. In Fig. 2, for each statistic, we plotted $\bar{\alpha}$ in the left panel, and $1-\bar{\beta}$ in the upper right panel for (i) and the lower right panel for (ii) in each case of (a) to (c). One can observe that $T_{\text {IYA }}$ gave preferable performances both for the size and power in (a) to (c). $\widetilde{T}_{n_{1}, n_{2}}$ gave bad performances compared with $T_{\text {IYA }}$ with respect to the power of (i) in (a) because $\boldsymbol{h}_{1(1)}=\boldsymbol{h}_{1(2)}$ and (2.11) holds for (i) in (a). See the last paragraph in Section 4. On the other hand, $T_{V}$ gave bad performances for (b) and (c) with respect to the size. This is probably 
because the bias of $1-\min \left\{1,\left(\tilde{\boldsymbol{h}}_{1(1)}^{T} \tilde{\boldsymbol{h}}_{1(2)}\right)^{2}\right\}$ is quite large for (b) and (c). See (3.4). As for $T_{\mathrm{LC}}$, it gave bad performances with respect to the size in (a) and (b). Remember that $T_{\mathrm{LC}}$ was constructed under (1.2).
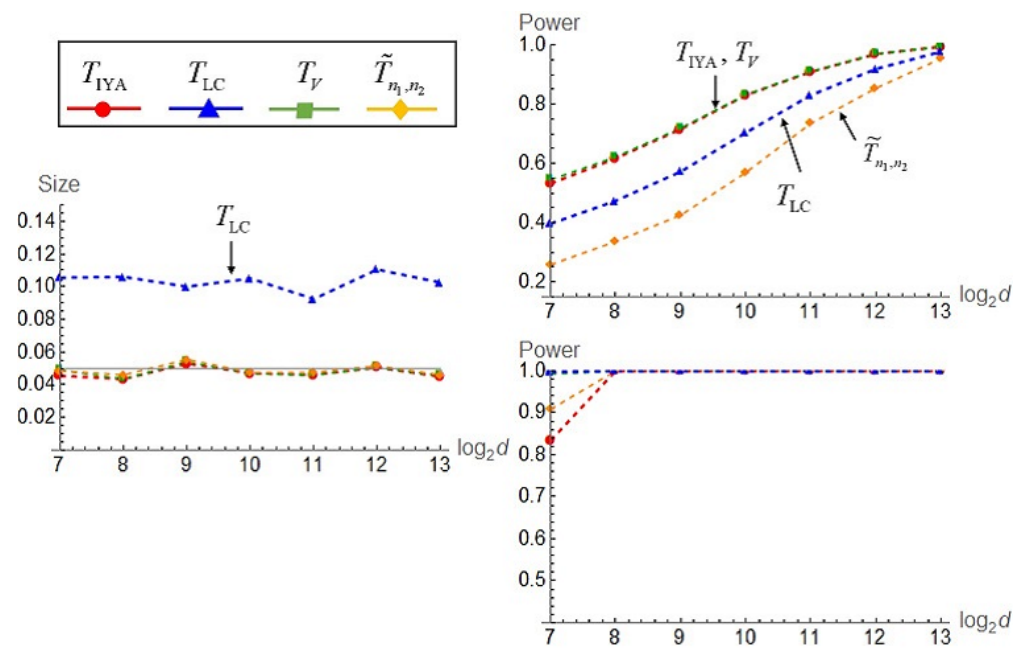

(a) When $\alpha_{1}=1, \alpha_{2}=1 / 3, c_{1}=1$ and $\theta=\pi / 6$. In this case, (1.3) and (A-ii) hold. Note that $\lambda_{1(i)} / \lambda_{2(i)} \approx d^{2 / 3}$ for $i=1,2$.
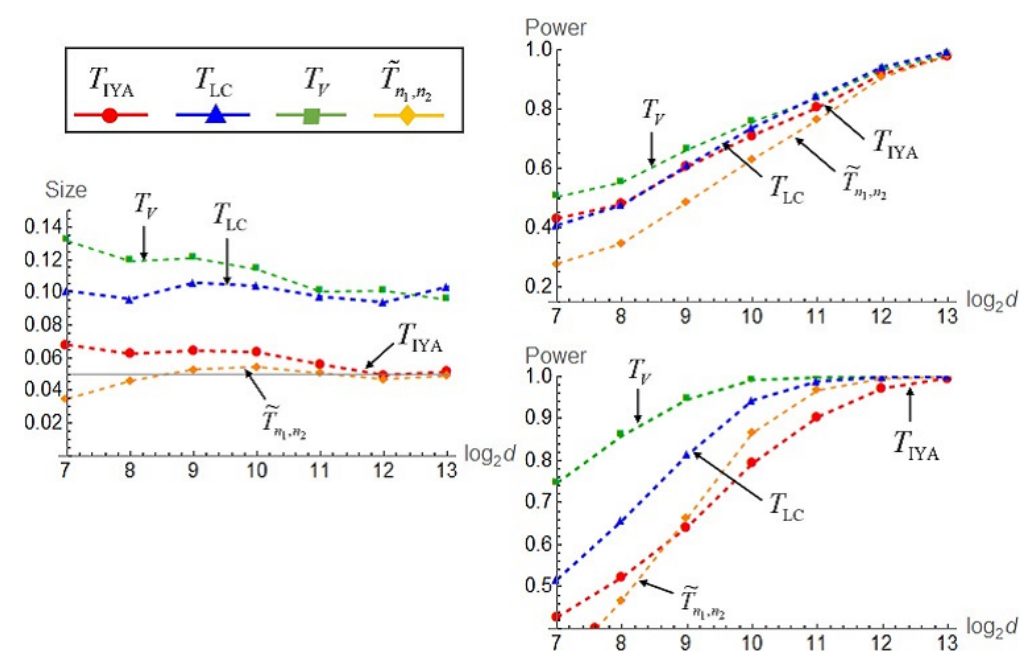

(b) When $\alpha_{1}=1, \alpha_{2}=1, c_{1}=1 / 3$ and $\theta=\pi / 6$. In this case, (A-ii) holds, while (1.3) does not hold. Note that $\lambda_{1(i)} / \lambda_{2(i)} \approx 3$ for $i=1,2$. 


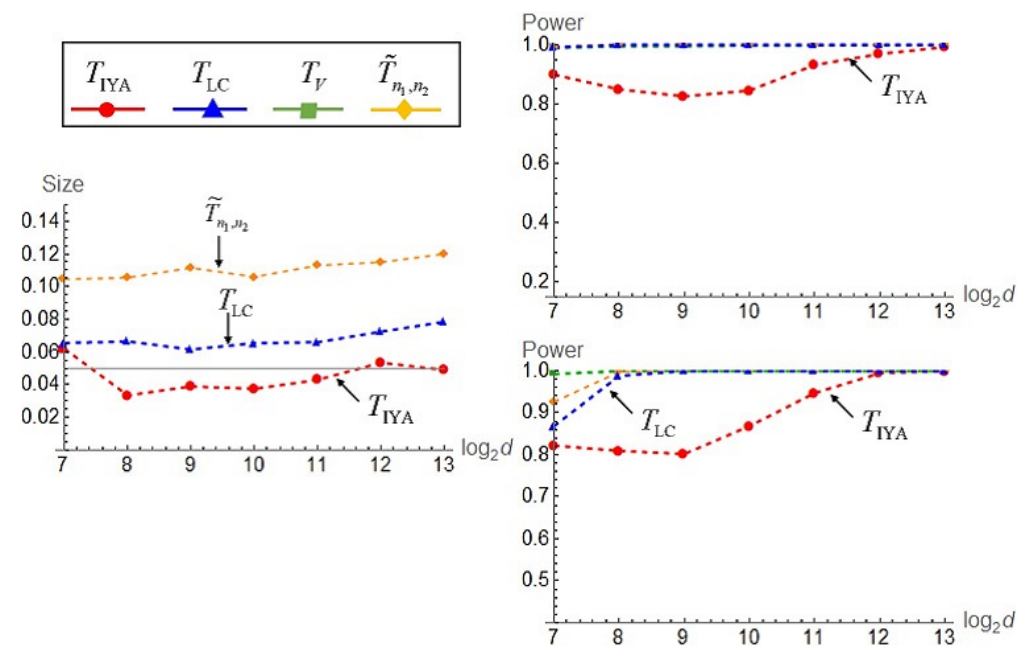

(c) When $\alpha_{1}=1 / 2, \alpha_{2}=1 / 3, c_{1}=1$ and $\theta=\pi / 4$. In this case, (A-ii) holds, while (1.3) does not hold. Note that $\lambda_{1(i)} / \lambda_{2(i)} \approx d^{1 / 6}$ for $i=1,2$.

Figure 2. The performance of the test statistics, $T_{\mathrm{LC}}, \widetilde{T}_{n_{1}, n_{2}}, T_{V}$ and $T_{\mathrm{IYA}}$. We set $\left(n_{1}, n_{2}\right)=\left(3\left\lceil d^{1 / 2}\right\rceil, 4\left\lceil d^{1 / 2}\right\rceil\right)$ and $d=2^{s}$ for $s=7, \ldots, 13$. The value of $\bar{\alpha}$ is denoted by the dashed line in the left panel and the value of $1-\bar{\beta}$ is denoted by the dashed line in the upper right panel for (i) and in the lower right panel for (ii) for each statistic in each case of (a) to (c). In (c), $\bar{\alpha}$ of $T_{V}$ was too high to describe.

Next, we considered non-Gaussian distributions for $\pi_{i}, i=1,2$. We generated $\boldsymbol{x}_{i j}-\boldsymbol{\mu}_{i}\left(=\boldsymbol{H}_{i} \boldsymbol{\Lambda}_{i}^{1 / 2}\left(z_{1 j(i)}, \ldots, z_{d j(i)}\right)^{T}\right), j=1,2, \ldots,(i=1,2)$ independently from the following non-Gaussian distributions:

(I) $z_{1 j(i)}$ s are i.i.d. as $N(0,1)$ and $\left(z_{2 j(i)}, \ldots, z_{d j(i)}\right)^{T}$ s are i.i.d. as $(d-1)$-variate $t$-distribution, $t_{d-1}\left(\boldsymbol{I}_{d-1}, \nu\right)$, with mean zero, covariance matrix $\boldsymbol{I}_{d-1}$ and degrees of freedom $\nu=10$, where $z_{1 j(i)}$ and $\left(z_{2 j(i)}, \ldots, z_{d j(i)}\right)^{T}$ are independent; and

(II) $z_{1 j(i)}$ s are i.i.d. as $N(0,1)$ and $z_{s j(i)}=\left(v_{s j(i)}-5\right) / 10^{1 / 2}(s=2, \ldots, d)$ in which $v_{s j(i)} \mathrm{S}$ are i.i.d. as the chi-squared distribution with 5 degrees of freedom, where $z_{1 j(i)}$ and $\left(z_{2 j(i)}, \ldots, z_{d j(i)}\right)^{T}$ are independent.

Note that (A-i) holds for (II), however, does not for (I). As for (I), the second and third conditions in (A-i) are met while the first condition " $E\left(z_{q j(i)}^{2} z_{s j(i)}^{2}\right)=1$ " is not met from the fact that $E\left(z_{q j(i)}^{2} z_{s j(i)}^{2}\right)=1+O\left(\nu^{-1}\right)$ for all $q \neq s(\geq 2)$. Also, 
note that (A-iii) holds both for (I) and (II). In (5.1), instead of (5.2), we set

$$
\boldsymbol{\Sigma}_{1(i)}=\gamma_{i}\left(\begin{array}{cc}
\boldsymbol{\Gamma}_{d_{1(i)}} & \boldsymbol{O} \\
\boldsymbol{O} & \boldsymbol{\Gamma}_{d_{2(i)}}
\end{array}\right) \text { and } \boldsymbol{\Sigma}_{2(1)}=\left(0.2^{|i-j|^{1 / 3}}\right)
$$

where $\boldsymbol{\Gamma}_{s}=\left(\boldsymbol{I}_{s}+\mathbf{1}_{s} \mathbf{1}_{s}^{T}\right) / 2$ with $\mathbf{1}_{s}=(1, \ldots, 1)^{T}, \gamma_{1}=1, d_{1(1)}=\left\lceil 2 d^{\alpha_{1}}\right\rceil$ and $d_{2(1)}=\left\lceil 2 d^{\alpha_{1}} / 3\right\rceil$. Note that $\lambda_{1(1)} \approx d^{\alpha_{1}}$ and $\lambda_{2(1)} / \lambda_{1(1)} \approx 1 / 3$. As for the alternative hypothesis, we considered two cases:

(iii) $\boldsymbol{\Sigma}_{1(2)}=1.5 \boldsymbol{\Sigma}_{1(1)}$ and $\boldsymbol{\Sigma}_{2(2)}=1.5\left(0.4^{|i-j|^{1 / 3}}\right)$; and

(iv) $\gamma_{2}=1, d_{1(2)}=\left\lceil(4 / 3) d^{\alpha_{1}}\right\rceil, d_{2(2)}=d_{2(1)}$ and $\Sigma_{2(2)}=\left(0.4^{|i-j|^{1 / 3}}\right)$.

Note that $\lambda_{1(1)} / \lambda_{1(2)}=2 / 3, \boldsymbol{h}_{1(1)}^{T} \boldsymbol{h}_{1(2)}=1, \kappa_{1} \neq \kappa_{2}$ and $\delta_{1} \neq \delta_{2}$ in (iii), and $\lambda_{1(1)} / \lambda_{1(2)} \approx 3 / 2, \boldsymbol{h}_{1(1)}^{T} \boldsymbol{h}_{1(2)} \neq 1, \kappa_{1} \neq \kappa_{2}$ and $\delta_{1} \neq \delta_{2}$ in (iv). We considered two cases:

(d) $\alpha_{1}=4 / 5$ in (I) and (e) $\alpha_{1}=1 / 2$ in (II).

Note that for (d) and (e) (A-ii) holds, while (1.3) does not hold.

Similar to Fig. 2, we calculated $\bar{\alpha}$ and $1-\bar{\beta}$ by 2000 replications and plotted the results in Fig. 3. In Fig. 3, one can observe that $T_{\text {IYA }}$ gave adequate performances for (d) as $d$ and $n_{i} \mathrm{~s}$ increase even when (A-i) is not met. For (e), $T_{\text {IYA }}$ performed well both for the size and power as $d$ and $n_{i}$ s increase.

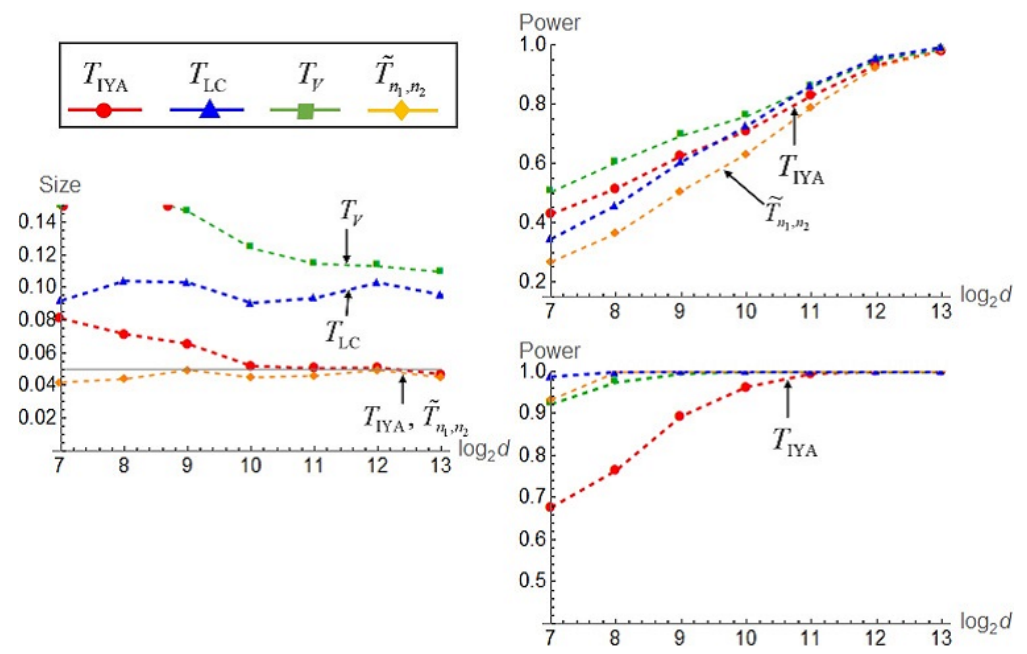

(d) When $\alpha_{1}=4 / 5$. In this case, (A-ii) holds, while (A-i) does not hold. Note that $\lambda_{1(1)} / \lambda_{2(1)} \approx 3$ and $\lambda_{1(2)} / \lambda_{2(2)} \approx 3$ for (iii), and $\lambda_{1(2)} / \lambda_{2(2)} \approx 2$ for (iv). 


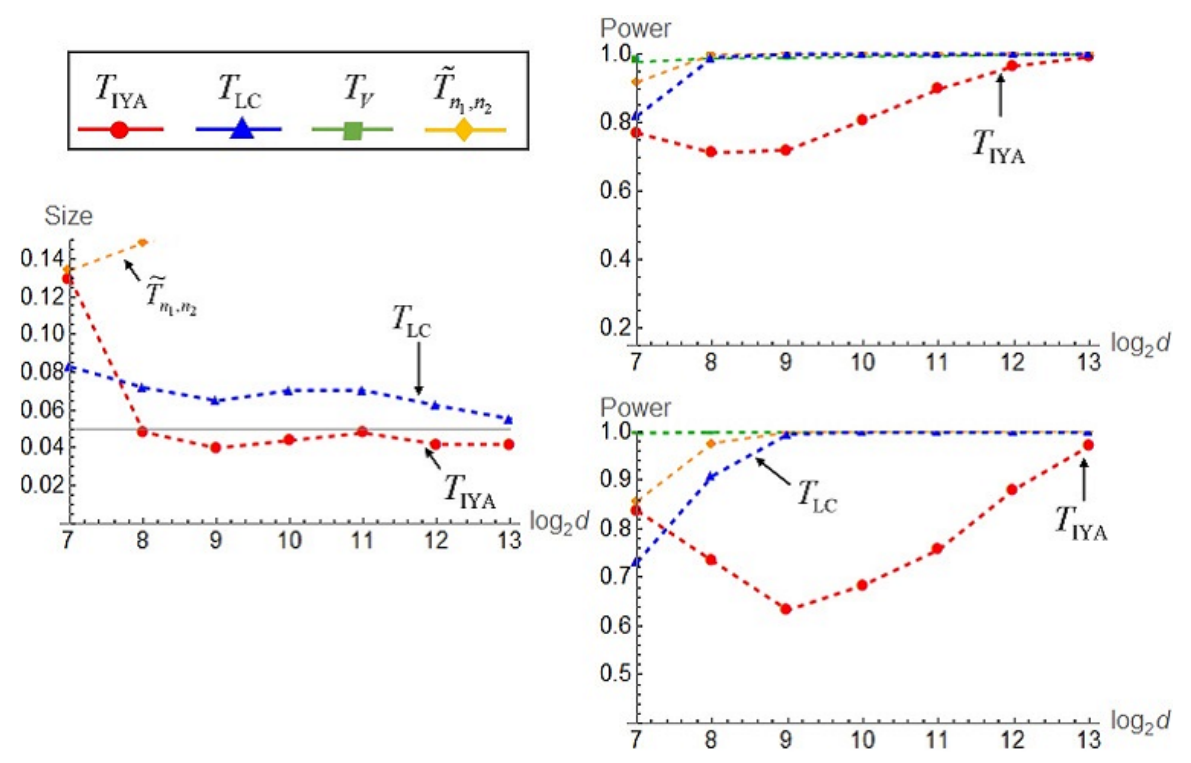

(e) When $\alpha_{1}=1 / 2$. In this case, (A-i) and (A-ii) hold. Note that $\lambda_{1(1)} / \lambda_{2(1)} \approx 3$ and $\lambda_{1(2)} / \lambda_{2(2)} \approx 3$ for (iii), and $\lambda_{1(2)} / \lambda_{2(2)} \approx 2$ for (iv).

Figure 3. The performance of the test statistics, $T_{\mathrm{LC}}, \widetilde{T}_{n_{1}, n_{2}}, T_{V}$ and $T_{\mathrm{IYA}}$. We set $\left(n_{1}, n_{2}\right)=\left(3\left\lceil d^{1 / 2}\right\rceil, 4\left\lceil d^{1 / 2}\right\rceil\right)$ and $d=2^{s}$ for $s=7, \ldots, 13$. The value of $\bar{\alpha}$ is denoted by the dashed line in the left panel and the value of $1-\bar{\beta}$ is denoted by the dashed line in the upper right panel for (iii) and the lower right panel for (iv) for each statistic in each case of (d) and (e). In (e), $\bar{\alpha}$ of $T_{V}$ was too high to describe.

\section{Data analysis}

We analyzed gene expression data by using the proposed test procedure based on $T_{\text {IYA }}$. We handled microarray data of Glioma with $54613(=d)$ probes consisting of four classes: oligodendroglioma (ODG) (50 samples), glioblastoma (GBT) (81 samples), astrocytoma (AT) (26 samples) and non-tumor (NT) (23 samples). See Sun et al. (2006) for the details. The data set is available at NCBI Gene Expression Omnibus. First, we checked the condition (A-ii) by applying a method given in Section 5 of Aoshima and Yata (2018) and confirmed that each class fits the SSE model. According to the proposed test procedure given in Section 4 with 


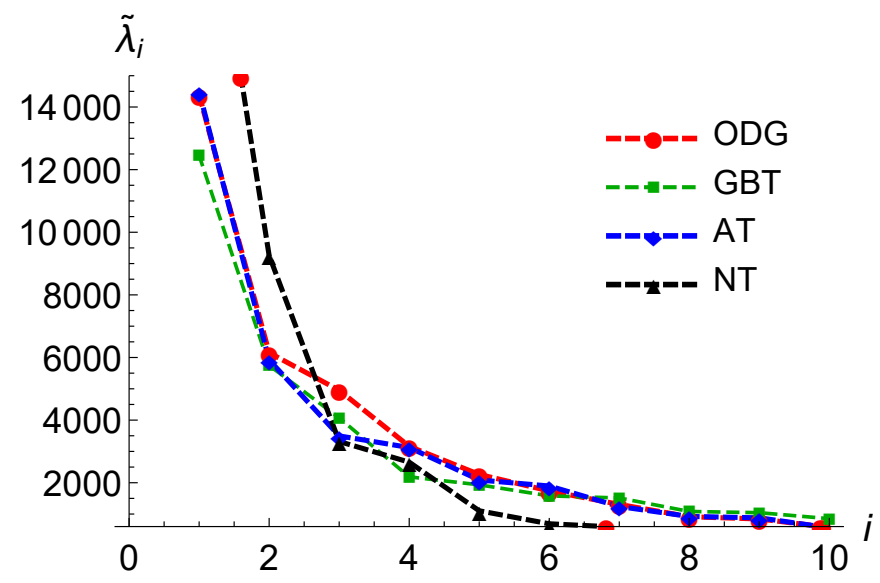

Figure 4. Estimates of the first ten eigenvalues by using the NR method for each class in Sun et al. (2006)'s dataset.

$T_{\text {IYA }}$, we tested (1.1) at significant level 0.05 . Then, $H_{0}$ was rejected for (ODG, AT), (ODG, NT), (GBT, NT) and (AT, NT). The results were summarized in Table 1.

In addition, we investigated eigenvalues of each class. We standardized each sample by $\boldsymbol{x}_{i j} /\left\{\operatorname{tr}\left(\boldsymbol{S}_{i n_{i}}\right) / d\right\}^{1 / 2}$ for all $i, j$ so as to have $\operatorname{tr}\left(\boldsymbol{S}_{i n_{i}}\right)\left(=\sum_{s=1}^{n_{i}-1} \hat{\lambda}_{s(i)}\right)=$ $d$. We calculated the first ten eigenvalues by using the NR method. In Fig. 4, we plotted them for each class. One can observe that the first eigenvalues are strongly spiked for each class.

Table 1. The values of $T_{\mathrm{IYA}}$ and P-values for all pairs of the data sets in Sun et al. (2006). Each data set is of $d=54613$.

\begin{tabular}{c|cccccc}
\hline & $(\mathrm{ODG}, \mathrm{GBT})$ & $(\mathrm{ODG}, \mathrm{AT})$ & $(\mathrm{ODG}, \mathrm{NT})$ & $(\mathrm{GBT}, \mathrm{AT})$ & $(\mathrm{GBT}, \mathrm{NT})$ & $(\mathrm{AT}, \mathrm{NT})$ \\
\hline$\left(n_{1}, n_{2}\right)$ & $(50,81)$ & $(50,26)$ & $(50,23)$ & $(81,26)$ & $(81,23)$ & $(26,23)$ \\
$T_{\text {IYA }}$ & 3.63 & 6.81 & 44 & 2.61 & 102 & 40.60 \\
P-value & 0.056 & 0.009 & 0 & 0.106 & 0 & 0 \\
\hline
\end{tabular}

\section{Conclusion}

It is quite important to select a suitable test procedure which fits the eigenstructure. Any eigenstructure is classified into the SSE model or the NSSE model, 
which are distinguished by a method of Aoshima and Yata (2018). When the data fits the NSSE model (1.2), one may use $T_{\mathrm{LC}}$ given by Li and Chen (2012). On the other hand, when (1.3) is met, one may use $\widetilde{T}_{n_{1}, n_{2}}$ given in Section 2.2. However, it should be noted that $\widetilde{T}_{n_{1}, n_{2}}$ cannot distinguish two covariance matrices when they share the first eigenspace. It is because under (1.3) the strongly-spiked first eigenvalue obscures the difference between $\Sigma_{1 \star}$ and $\Sigma_{2 \star}$. (1.3) is one of the SSE models and it is sometimes rather strict in actual data analyses. In order to overcome the difficulties, we created $T_{\mathrm{IYA}}$ and gave a test procedure by $T_{\mathrm{IYA}}$ under the SSE model (A-ii) even when (1.3) is not met. Since $T_{\text {IYA }}$ utilizes the difference of not only the first eigenspaces but also the remaining eigenspaces, the proposed test procedure based on $T_{\mathrm{IYA}}$ can successfully detect the disparity between the two covariance matrices. In conclusion, we recommend using $T_{\mathrm{IYA}}$ when the data fits the SSE model (A-ii).

\section{Appendix A. Estimation of several parameters in the new test procedure}

In this section, we give asymptotic properties of the estimators for the parameters in the new test procedure.

Appendix A.1. Estimation of $\lambda_{1(i)}$ and $\boldsymbol{h}_{1(i)}$

Let $s_{1(i)}=\sum_{j=1}^{n_{i}}\left(z_{1 j(i)}-\bar{z}_{1(i)}\right)^{2} /\left(n_{i}-1\right)$ for $i=1,2$, where $\bar{z}_{1(i)}=n_{i}^{-1} \sum_{j=1}^{n_{i}} z_{1 j(i)}$.

Lemma A.1. Under $(A-i)$ and (A-ii), it holds that as $m \rightarrow \infty$

$$
\begin{aligned}
\frac{\tilde{\lambda}_{1(i)}}{\lambda_{1(i)}}=s_{1(i)} & +O_{p}\left(\delta_{i}^{1 / 2} /\left(n_{i} \lambda_{1(i)}\right)\right)=1+o_{p}(1) \text { for } i=1,2 \text {, and } \\
\left|\tilde{\boldsymbol{h}}_{1(1)}^{T} \tilde{\boldsymbol{h}}_{1(2)}\right|= & \left|\boldsymbol{h}_{1(1)}^{T} \boldsymbol{h}_{1(2)}\right|+O_{p}\left(\frac{\delta_{1}^{1 / 2}}{n_{1} \lambda_{1(1)}}+\frac{\delta_{2}^{1 / 2}}{n_{2} \lambda_{1(2)}}\right) \\
& +O_{p}\left(\left\{1-\left(\boldsymbol{h}_{1(1)}^{T} \boldsymbol{h}_{1(2)}\right)^{2}\right\}^{1 / 2} / n_{\text {min }}^{1 / 2}\right) \\
= & \left|\boldsymbol{h}_{1(1)}^{T} \boldsymbol{h}_{1(2)}\right|+o_{p}(1) .
\end{aligned}
$$

In addition, under (A-i) to (A-iii), it holds that as $m \rightarrow \infty$

$$
\sqrt{\frac{n_{i}-1}{2}}\left(\frac{\tilde{\lambda}_{1(i)}}{\lambda_{1(i)}}-1\right) \Rightarrow N(0,1) .
$$


On the other hand, from (B.10) in Appendix B of the online supplementary, it holds that as $m \rightarrow \infty$

$$
\hat{\lambda}_{1(i)} / \lambda_{1(i)}=1+\kappa_{i} /\left(n_{i} \lambda_{1(i)}\right)+o_{p}(1)
$$

under (A-i) and (A-ii). If $\liminf \operatorname{in}_{m \rightarrow \infty} \kappa_{i} /\left(n_{i} \lambda_{1(i)}\right)>0, \hat{\lambda}_{1(i)}$ and $\hat{\boldsymbol{h}}_{1(i)}$ have inconsistency properties in the sense that $\hat{\lambda}_{1(i)} / \lambda_{1(i)}>1+o_{p}(1)$ and $\left|\hat{\boldsymbol{h}}_{1(1)}^{T} \hat{\boldsymbol{h}}_{1(2)}\right|<$ $\left|\boldsymbol{h}_{1(1)}^{T} \boldsymbol{h}_{1(2)}\right|+o_{p}(1)$ from the fact that $\hat{\boldsymbol{h}}_{1(i)}=\left(\tilde{\lambda}_{1(i)} / \hat{\lambda}_{1(i)}\right)^{1 / 2} \tilde{\boldsymbol{h}}_{1(i)}$. However, $\tilde{\lambda}_{1(i)}$ and $\tilde{\boldsymbol{h}}_{1(1)}$ have the consistency properties even when $\liminf _{m \rightarrow \infty} \kappa_{i} /\left(n_{i} \lambda_{1(i)}\right)>0$.

Appendix A.2. Estimation of $\delta_{i}$ and $\kappa_{i}$

First, we consider estimation of $\delta_{i}$. Aoshima and Yata (2018) gave an estimator of $\delta_{i}$ by using the CDM method: Let $n_{i 1}=\left\lceil n_{i} / 2\right\rceil$ and $n_{i 2}=n_{i}-n_{i 1}$. Let $\boldsymbol{X}_{i 1}=\left[\boldsymbol{x}_{i 1}, \ldots, \boldsymbol{x}_{i n_{i 1}}\right]$ and $\boldsymbol{X}_{i 2}=\left[\boldsymbol{x}_{i n_{i 1}+1}, \ldots, \boldsymbol{x}_{i n_{i}}\right]$. We define

$$
\boldsymbol{S}_{i C}=\left\{\left(n_{i 1}-1\right)\left(n_{i 2}-1\right)\right\}^{-1 / 2}\left(\boldsymbol{X}_{i 1}-\overline{\boldsymbol{X}}_{i 1}\right)^{T}\left(\boldsymbol{X}_{i 2}-\overline{\boldsymbol{X}}_{i 2}\right),
$$

where $\overline{\boldsymbol{X}}_{i j}=\left[\overline{\boldsymbol{x}}_{i j}, \ldots, \overline{\boldsymbol{x}}_{i j}\right]$ with $\overline{\boldsymbol{x}}_{i 1}=\sum_{l=1}^{n_{i 1}} \boldsymbol{x}_{i l} / n_{i 1}$ and $\overline{\boldsymbol{x}}_{i 2}=\sum_{l=n_{i 1}+1}^{n_{i}} \boldsymbol{x}_{i l} / n_{i 2}$. We estimate $\lambda_{j(i)}$ by the $j$-th singular value, $\hat{\lambda}_{j(i)}$, of $\boldsymbol{S}_{i C}$, where $\hat{\lambda}_{1(i)} \geq \cdots \geq$ $\hat{\lambda}_{n_{i(2)}-1(i)} \geq 0$. Also, we estimate $\operatorname{tr}\left(\boldsymbol{\Sigma}_{i}^{2}\right)$ by $\operatorname{tr}\left(\boldsymbol{S}_{i C} \boldsymbol{S}_{i C}^{T}\right)$. Note that $E\left\{\operatorname{tr}\left(\boldsymbol{S}_{i C} \boldsymbol{S}_{i C}^{T}\right)\right\}=$ $\operatorname{tr}\left(\Sigma_{i}^{2}\right)$. Finally, $\delta_{i}$ is estimated by

$$
\hat{\delta}_{i}=\operatorname{tr}\left(\boldsymbol{S}_{i C} \boldsymbol{S}_{i C}^{T}\right)-\hat{\lambda}_{1(i)}^{2} .
$$

We have the following result.

Lemma A.2. Under (A-i) and (A-ii), it holds that $\hat{\delta}_{i} / \delta_{i}=1+o_{p}(1)$ as $m \rightarrow \infty$ for $i=1,2$.

Next, for $\hat{\kappa}_{i}$, we have the following result.

Lemma A.3. Under (A-i) and (A-ii), it holds that $\hat{\kappa}_{i} / \kappa_{i}=1+o_{p}(1)$ as $m \rightarrow \infty$ for $i=1,2$.

\section{Proofs}

We provide all proofs of the theoretical results given in this paper in Appendix B of the online supplementary. 


\section{Acknowledgements}

We would like to thank the editor and two anonymous referees for their constructive comments. Research of the first author was partially supported by Grantin-Aid for Young Scientists, Japan Society for the Promotion of Science (JSPS), under Contract Number 18K18015. Research of the second author was partially supported by Grant-in-Aid for Scientific Research (C), JSPS, under Contract Number 18K03409. Research of the third author was partially supported by Grants-inAid for Scientific Research (A) and Challenging Research (Exploratory), JSPS, under Contract Numbers $15 \mathrm{H} 01678$ and 17K19956.

\section{References}

Aoshima, M., Yata, K., 2011. Two-stage procedures for high-dimensional data. Sequential Anal. (Editor's special invited paper) 30, 356-399.

Aoshima, M., Yata, K., 2018. Two-sample tests for high-dimension, strongly spiked eigenvalue models. Stat. Sin. 28, 43-62.

Bai, Z., Saranadasa, H., 1996. Effect of high dimension: By an example of a two sample problem. Stat. Sin. 6, 311-329.

Chen, S.X., Qin, Y.-L., 2010. A two-sample test for high-dimensional data with applications to gene-set testing. Ann. Statist. 38, 808-835.

Ishii, A., Yata, K., Aoshima, M., 2016. Asymptotic properties of the first principal component and equality tests of covariance matrices in high-dimension, lowsample-size context. J. Stat. Plan. Inference 170, 186-199.

Ishii, A., 2017a. A two-sample test for high-dimension, low-sample-size data under the strongly spiked eigenvalue model. Hiroshima Math. J. 47, 273-288.

Ishii, A., 2017b. A high-dimensional two-sample test for non-Gaussian data under a strongly spiked eigenvalue model. J. Japan Statist. Soc. 47, 273-291.

Jung, S., Marron, J.S., 2009. PCA consistency in high dimension, low sample size context. Ann. Statist. 37, 4104-4130.

Li, J., Chen, S.X., 2012. Two sample tests for high-dimensional covariance matrices. Ann. Statist. 40, 908-940. 
Schott, J.R., 2007. A test for the equality of covariance matrices when the dimension is large relative to the sample sizes. Comput. Statist. Data Anal. 51, 6535-6542.

Shen, D., Shen, H., Zhu, H., Marron, J.S., 2016. The statistics and mathematics of high dimension low sample size asymptotics. Stat. Sin. 26, 1747-1770.

Srivastava, M.S., Yanagihara, H., 2010. Testing the equality of several covariance matrices with fewer observations than the dimension. J. Multivariate Anal. 101, 1319-1329.

Sun, L., Hui, A.M., Su, Q., Vortmeyer, A., Kotliarov, Y., Pastorino, S., Passaniti, A., Menon, J., Walling, J., Bailey, R., Rosenblum, M., Mikkelsen, T., Fine, H.A., 2006. Neuronal and glioma-derived stem cell factor induces angiogenesis within the brain. Cancer Cell 9, 287-300.

Yata, K., Aoshima, M., 2010. Effective PCA for high-dimension, low-samplesize data with singular value decomposition of cross data matrix. J. Multivariate Anal. 101, 2060-2077.

Yata, K., Aoshima, M., 2012. Effective PCA for high-dimension, low-sample-size data with noise reduction via geometric representations. J. Multivariate Anal. 105, 193-215.

Yata, K., Aoshima, M., 2013. PCA consistency for the power spiked model in high-dimensional settings. J. Multivariate Anal. 122, 334-354. 


\title{
Supplement to "Equality tests of high-dimensional covariance matrices under the strongly spiked eigenvalue model"
}

\author{
Aki Ishii \\ Department of Information Sciences \\ Tokyo University of Science \\ Kazuyoshi Yata \\ Institute of Mathematics \\ University of Tsukuba \\ Makoto Aoshima \\ Institute of Mathematics \\ University of Tsukuba
}

\begin{abstract}
In this supplement, we present technical details of the main work in Ishii et al. [3] together with an additional simulation. We give proofs of the theorems, corollaries, propositions and lemmas in the article. We also give an additional simulation result for Section 5 in the article. The equation numbers and the mathematical symbols used in the supplement are the same as those which are made reference to in the main document.
\end{abstract}

\section{Appendix B. Proofs}

Throughout this appendix, we assume $\boldsymbol{\mu}_{i}=\mathbf{0}, i=1,2$, without loss of generality. Let $\boldsymbol{\Sigma}_{1,2}=$ $\boldsymbol{\Sigma}_{1}-\boldsymbol{\Sigma}_{2}$ and $\xi=\left\{1-\left(\boldsymbol{h}_{1(1)}^{T} \boldsymbol{h}_{1(2)}\right)^{2}\right\}^{1 / 2}$.

Proof of Lemma 2.1. We assume (A-i) and (1.3). Note that as $m \rightarrow \infty$

$$
\operatorname{Var}\left(\sum_{j \neq k \neq l \neq l^{\prime}}^{n_{i}} \frac{\boldsymbol{x}_{i j}^{T} \boldsymbol{x}_{i k} \boldsymbol{x}_{i l}^{T} \boldsymbol{x}_{i l^{\prime}}}{n_{i}^{4}}\right)=O\left(\frac{\left\{\operatorname{tr}\left(\boldsymbol{\Sigma}_{i}^{2}\right)\right\}^{2}+\operatorname{tr}\left(\boldsymbol{\Sigma}_{i}^{4}\right)}{n_{i}^{4}}\right)=O\left(\frac{\lambda_{1(i)}^{4}}{n_{i}^{4}}\right)
$$

for $i=1,2$. Then, by using Chebyshev's inequality, it holds that

$$
\frac{1}{n_{i}\left(n_{i}-1\right)\left(n_{i}-2\right)\left(n_{i}-3\right)} \sum_{j \neq k \neq l \neq l^{\prime}}^{n_{i}} \boldsymbol{x}_{i j}^{T} \boldsymbol{x}_{i k} \boldsymbol{x}_{i l}^{T} \boldsymbol{x}_{i l^{\prime}}=o_{p}(K) \text { for } i=1,2 .
$$

Address correspondence to Makoto Aoshima, Institute of Mathematics, University of Tsukuba, Ibaraki 305-8571, Japan; Fax: +81-298-53-6501; E-mail: aoshima@math.tsukuba.ac.jp 
On the other hand, by noting that $\left|E\left\{\boldsymbol{x}_{i k}^{T}\left(\boldsymbol{x}_{i j} \boldsymbol{x}_{i j}^{T}-\boldsymbol{\Sigma}\right) \boldsymbol{x}_{i l} \boldsymbol{x}_{i l}^{T}\left(\boldsymbol{x}_{i k} \boldsymbol{x}_{i k}^{T}-\boldsymbol{\Sigma}\right) \boldsymbol{x}_{i j}\right\}\right| \leq E\left\{\left(\boldsymbol{x}_{i k}^{T}\left(\boldsymbol{x}_{i j} \boldsymbol{x}_{i j}^{T}-\right.\right.\right.$ $\left.\left.\boldsymbol{\Sigma}) \boldsymbol{x}_{i l}\right)^{2}\right\}$ for $j \neq k \neq l$, it holds that

$$
E\left\{\left(\frac{1}{n_{i}\left(n_{i}-1\right)\left(n_{i}-2\right)} \sum_{j \neq k \neq l}^{n_{i}} \boldsymbol{x}_{i k}^{T}\left(\boldsymbol{x}_{i j} \boldsymbol{x}_{i j}^{T}-\boldsymbol{\Sigma}_{i}\right) \boldsymbol{x}_{i l}\right)^{2}\right\}=O\left(\frac{\lambda_{1(i)}^{4}}{n_{i}^{3}}\right)=o\left(K^{2}\right)
$$

for $i=1,2$, so that

$$
\frac{1}{n_{i}\left(n_{i}-1\right)\left(n_{i}-2\right)} \sum_{j \neq k \neq l}^{n_{i}} \boldsymbol{x}_{i k}^{T} \boldsymbol{x}_{i j} \boldsymbol{x}_{i j}^{T} \boldsymbol{x}_{i l}=\frac{1}{n_{i}\left(n_{i}-1\right)} \sum_{k \neq l}^{n_{i}} \boldsymbol{x}_{i k}^{T} \boldsymbol{\Sigma}_{i} \boldsymbol{x}_{i l}+o_{p}(K)
$$

for $i=1,2$. By noting that $\operatorname{tr}\left\{\left(\boldsymbol{\Sigma}_{1} \boldsymbol{\Sigma}_{2}\right)^{2}\right\} \leq\left\{\operatorname{tr}\left(\boldsymbol{\Sigma}_{1} \boldsymbol{\Sigma}_{2}\right)\right\}^{2} \leq \operatorname{tr}\left(\boldsymbol{\Sigma}_{1}^{2}\right) \operatorname{tr}\left(\boldsymbol{\Sigma}_{2}^{2}\right)$, similar to (B.1) and (B.2), we have that

$$
\begin{aligned}
& \frac{1}{n_{1} n_{2}\left(n_{1}-1\right)\left(n_{2}-1\right)} \sum_{j \neq k}^{n_{1}} \sum_{l \neq l^{\prime}}^{n_{2}} \boldsymbol{x}_{1 j}^{T} \boldsymbol{x}_{2 l} \boldsymbol{x}_{1 k}^{T} \boldsymbol{x}_{2 l^{\prime}}=o_{p}(K) \text { and } \\
& \frac{1}{n_{1} n_{2}\left(n_{i}-1\right)} \sum_{j=1}^{n_{i^{\prime}}} \sum_{k \neq l}^{n_{i}} \boldsymbol{x}_{i k}^{T} \boldsymbol{x}_{i^{\prime} j} \boldsymbol{x}_{i^{\prime} j}^{T} \boldsymbol{x}_{i l}=\frac{1}{n_{i}\left(n_{i}-1\right)} \sum_{k \neq l}^{n_{i}} \boldsymbol{x}_{i k}^{T} \boldsymbol{\Sigma}_{i^{\prime}} \boldsymbol{x}_{i l}+o_{p}(K)
\end{aligned}
$$

for $i \neq i^{\prime}$. By noting that

$$
\operatorname{tr}\left(\boldsymbol{S}_{1 n_{1}} \boldsymbol{S}_{2 n_{2}}\right)=\sum_{j=1}^{n_{1}} \sum_{k=1}^{n_{2}} \frac{\left(\boldsymbol{x}_{1 j}^{T} \boldsymbol{x}_{2 k}\right)^{2}}{n_{1} n_{2}}-\sum_{i \neq i^{\prime}}^{2} \sum_{j=1}^{n_{i^{\prime}}} \sum_{k \neq l}^{n_{i}} \frac{\boldsymbol{x}_{i k}^{T} \boldsymbol{x}_{i^{\prime} j} \boldsymbol{x}_{i^{\prime} j}^{T} \boldsymbol{x}_{i l}}{n_{1} n_{2}\left(n_{i}-1\right)}+\sum_{j \neq k}^{n_{1}} \sum_{l \neq l^{\prime}}^{n_{2}} \frac{\boldsymbol{x}_{1 j}^{T} \boldsymbol{x}_{2 l} \boldsymbol{x}_{1 k}^{T} \boldsymbol{x}_{2 l^{\prime}}}{n_{1} n_{2}\left(n_{1}-1\right)\left(n_{2}-1\right)},
$$

we have from (B.1) to (B.3) that

$$
\begin{aligned}
U_{n_{1}, n_{2}}= & \sum_{i=1}^{2} \sum_{j \neq k}^{n_{i}} \frac{\left(\boldsymbol{x}_{i j}^{T} \boldsymbol{x}_{i k}\right)^{2}}{n_{i}\left(n_{i}-1\right)}-2 \sum_{j=1}^{n_{1}} \sum_{k=1}^{n_{2}} \frac{\left(\boldsymbol{x}_{1 j}^{T} \boldsymbol{x}_{2 k}\right)^{2}}{n_{1} n_{2}} \\
& +2 \sum_{i=1}^{2}(-1)^{i} \sum_{j \neq k}^{n_{i}} \frac{\boldsymbol{x}_{i j}^{T} \boldsymbol{\Sigma}_{1,2} \boldsymbol{x}_{i k}}{n_{i}\left(n_{i}-1\right)}+o_{p}(K) \\
= & \Delta+\sum_{i=1}^{2} \sum_{j \neq k}^{n_{i}} \frac{\operatorname{tr}\left\{\left(\boldsymbol{x}_{i j} \boldsymbol{x}_{i j}^{T}-\boldsymbol{\Sigma}_{i}\right)\left(\boldsymbol{x}_{i k} \boldsymbol{x}_{i k}^{T}-\boldsymbol{\Sigma}_{i}\right)\right\}}{n_{i}\left(n_{i}-1\right)} \\
& -2 \sum_{j=1}^{n_{1}} \sum_{k=1}^{n_{2}} \frac{\operatorname{tr}\left\{\left(\boldsymbol{x}_{1 j} \boldsymbol{x}_{1 j}^{T}-\boldsymbol{\Sigma}_{1}\right)\left(\boldsymbol{x}_{2 k} \boldsymbol{x}_{2 k}^{T}-\boldsymbol{\Sigma}_{2}\right)\right\}}{n_{1} n_{2}} \\
& +2 \sum_{i=1}^{2}(-1)^{i} \sum_{j \neq k}^{n_{i}} \frac{\boldsymbol{x}_{i j}^{T} \boldsymbol{\Sigma}_{1,2} \boldsymbol{x}_{i k}}{n_{i}\left(n_{i}-1\right)}+2 \sum_{i=1}^{2}(-1)^{i+1} \sum_{j=1}^{n_{i}} \frac{\operatorname{tr}\left\{\left(\boldsymbol{x}_{i j} \boldsymbol{x}_{i j}^{T}-\boldsymbol{\Sigma}_{i}\right) \boldsymbol{\Sigma}_{1,2}\right\}}{n_{i}} \\
& +o_{p}(K) .
\end{aligned}
$$

Note that

$$
\sum_{s=2}^{d}\left(\boldsymbol{h}_{i(s)}^{T} \boldsymbol{\Sigma}_{1,2} \boldsymbol{h}_{i(s)}\right)^{2} \leq \sum_{s, t=2}^{d}\left(\boldsymbol{h}_{i(s)}^{T} \boldsymbol{\Sigma}_{1,2} \boldsymbol{h}_{i(t)}\right)^{2}=\operatorname{tr}\left\{\left(\boldsymbol{\Sigma}_{i \star} \boldsymbol{\Sigma}_{1,2}\right)^{2}\right\} \leq \lambda_{2(i)}^{2} \Delta
$$


and $\operatorname{tr}\left(\boldsymbol{\Sigma}_{i \star} \boldsymbol{\Sigma}_{1,2} \boldsymbol{\Sigma}_{i} \boldsymbol{\Sigma}_{1,2}\right) \leq \lambda_{1(i)} \lambda_{2(i)} \Delta$. Also, note that $\lambda_{2(i)} /\left(n_{i} K_{i}\right)^{1 / 2}=o(1)$ under (1.3). Let $\boldsymbol{y}_{i j}=\boldsymbol{x}_{i j}-\lambda_{1(i)}^{1 / 2} \boldsymbol{h}_{1(i)} z_{1 j(i)}$ for all $i, j$. Then, it holds that

$$
\begin{aligned}
& \operatorname{Var}\left(\sum_{j=1}^{n_{i}} \frac{\operatorname{tr}\left\{\left(\boldsymbol{y}_{i j} \boldsymbol{y}_{i j}^{T}-\boldsymbol{\Sigma}_{i \star}\right) \boldsymbol{\Sigma}_{1,2}\right\}}{n_{i}}\right)=O\left(\frac{\lambda_{2(i)}^{2} \Delta}{n_{i}}\right)=o(K \Delta) \\
& \text { and } \operatorname{Var}\left(\sum_{j=1}^{n_{i}} \frac{\operatorname{tr}\left\{\left(\boldsymbol{y}_{i j} \boldsymbol{x}_{i j}^{T}-\boldsymbol{\Sigma}_{i \star}\right) \boldsymbol{\Sigma}_{1,2}\right\}}{n_{i}}\right)=o(K \Delta)
\end{aligned}
$$

for $i=1,2$, so that

$$
\begin{aligned}
& (-1)^{i+1} \sum_{j=1}^{n_{i}} \frac{\operatorname{tr}\left\{\left(\boldsymbol{x}_{i j} \boldsymbol{x}_{i j}^{T}-\boldsymbol{\Sigma}_{i}\right) \boldsymbol{\Sigma}_{1,2}\right\}}{n_{i}} \\
& =\sum_{j=1}^{n_{i}} \frac{\lambda_{1(i)}\left(z_{1 j(i)}^{2}-1\right)\left\{\lambda_{1(i)}-\lambda_{1\left(i^{\prime}\right)}\left(\boldsymbol{h}_{1(1)}^{T} \boldsymbol{h}_{1(2)}\right)^{2}\right\}}{n_{i}}+o_{p}\left\{(K \Delta)^{1 / 2}\right\}
\end{aligned}
$$

for $i=1,2 ; i^{\prime} \neq i$. Note that

$$
\sum_{j=1}^{n_{i}} \frac{\lambda_{1(1)} \lambda_{1(2)}\left(z_{1 j(i)}^{2}-1\right)\left\{1-\left(\boldsymbol{h}_{1(1)}^{T} \boldsymbol{h}_{1(2)}\right)^{2}\right\}}{n_{i}}=O_{p}\left(n_{\max }^{1 / 2} K \xi^{2}\right)
$$

from the fact that $\lambda_{1(1)}^{2} \lambda_{1(2)}^{2} /\left(n_{1} n_{2}\right)=O\left(K^{2}\right)$. Then, from (B.5), it follows

$$
\begin{aligned}
& (-1)^{i+1} \sum_{j=1}^{n_{i}} \frac{\operatorname{tr}\left\{\left(\boldsymbol{x}_{i j} \boldsymbol{x}_{i j}^{T}-\boldsymbol{\Sigma}_{i}\right) \boldsymbol{\Sigma}_{1,2}\right\}}{n_{i}} \\
& =\left(\lambda_{1(i)}-\lambda_{1\left(i^{\prime}\right)}\right) \sum_{j=1}^{n_{i}} \frac{\lambda_{1(i)}\left(z_{1 j(i)}^{2}-1\right)}{n_{i}}+o_{p}\left\{(K \Delta)^{1 / 2}\right\}+O_{p}\left(n_{\max }^{1 / 2} K \xi^{2}\right) .
\end{aligned}
$$

Similar to (B.5), we have that

$$
\sum_{j \neq k}^{n_{i}} \frac{\boldsymbol{x}_{i j}^{T} \boldsymbol{\Sigma}_{1,2} \boldsymbol{x}_{i k}}{n_{i}\left(n_{i}-1\right)}=O_{p}\left(\frac{\left[\operatorname{tr}\left\{\left(\boldsymbol{\Sigma}_{i} \boldsymbol{\Sigma}_{1,2}\right)^{2}\right\}\right]^{1 / 2}}{n_{i}}\right)=O_{p}\left(\frac{\lambda_{1(i)} \Delta^{1 / 2}}{n_{i}}\right)=o_{p}\left\{(K \Delta)^{1 / 2}\right\}
$$

for $i=1,2$. On the other hand, we have that

$$
\begin{aligned}
\operatorname{Var}\left(\sum_{j \neq k}^{n_{i}} \frac{\operatorname{tr}\left\{\left(\boldsymbol{y}_{i j} \boldsymbol{y}_{i j}^{T}-\boldsymbol{\Sigma}_{i \star}\right)\left(\boldsymbol{x}_{i k} \boldsymbol{x}_{i k}^{T}-\boldsymbol{\Sigma}_{i}\right)\right\}}{n_{i}^{2}}\right) & =O\left(\frac{\left\{\operatorname{tr}\left(\boldsymbol{\Sigma}_{i} \boldsymbol{\Sigma}_{i \star}\right)\right\}^{2}}{n_{i}^{2}}\right)=o\left(K^{2}\right) \\
\text { and } \operatorname{Var}\left(\sum_{j \neq k}^{n_{i}} \frac{\operatorname{tr}\left\{\left(\boldsymbol{y}_{i j} \boldsymbol{x}_{i j}^{T}-\boldsymbol{\Sigma}_{i \star}\right)\left(\boldsymbol{x}_{i k} \boldsymbol{x}_{i k}^{T}-\boldsymbol{\Sigma}_{i}\right)\right\}}{n_{i}^{2}}\right) & =o\left(K^{2}\right)
\end{aligned}
$$

for $i=1,2$. Then, it holds that

$$
\sum_{j \neq k}^{n_{i}} \frac{\operatorname{tr}\left\{\left(\boldsymbol{x}_{i j} \boldsymbol{x}_{i j}^{T}-\boldsymbol{\Sigma}_{i}\right)\left(\boldsymbol{x}_{i k} \boldsymbol{x}_{i k}^{T}-\boldsymbol{\Sigma}_{i}\right)\right\}}{n_{i}\left(n_{i}-1\right)}=\sum_{j \neq k}^{n_{i}} \frac{\lambda_{i(1)}^{2}\left(z_{1 j(1)}^{2}-1\right)\left(z_{1 k(1)}^{2}-1\right)}{n_{i}\left(n_{i}-1\right)}+o_{p}(K)
$$


for $i=1,2$. Similarly, we have that

$$
\begin{aligned}
& \sum_{j=1}^{n_{1}} \sum_{k=1}^{n_{2}} \frac{\operatorname{tr}\left\{\left(\boldsymbol{x}_{1 j} \boldsymbol{x}_{1 j}^{T}-\boldsymbol{\Sigma}_{1}\right)\left(\boldsymbol{x}_{2 k} \boldsymbol{x}_{2 k}^{T}-\boldsymbol{\Sigma}_{2}\right)\right\}}{n_{1} n_{2}} \\
& =\sum_{j=1}^{n_{1}} \sum_{k=1}^{n_{2}} \frac{\lambda_{1(1)} \lambda_{1(2)}\left(z_{1 j(1)}^{2}-1\right)\left(z_{1 k(2)}^{2}-1\right)\left(\boldsymbol{h}_{1(1)}^{T} \boldsymbol{h}_{1(2)}\right)^{2}}{n_{1} n_{2}}+o_{p}(K) .
\end{aligned}
$$

Note that

$$
\sum_{j=1}^{n_{1}} \sum_{k=1}^{n_{2}} \frac{\lambda_{1(1)} \lambda_{1(2)}\left(z_{1 j(1)}^{2}-1\right)\left(z_{1 k(2)}^{2}-1\right)\left\{1-\left(\boldsymbol{h}_{1(1)}^{T} \boldsymbol{h}_{1(2)}\right)^{2}\right\}}{n_{1} n_{2}}=O_{p}\left(K \xi^{2}\right) .
$$

Then, by combining (B.6) to (B.7) with (B.4), we have that

$$
\begin{aligned}
U_{n_{1}, n_{2}}= & \Delta_{1}+\left(W+\lambda_{1(1)}-\lambda_{1(2)}\right)^{2} \\
& -\sum_{i=1}^{2}\left\{\sum_{j=1}^{n_{1}} \frac{\lambda_{1(i)}^{2}\left(z_{1 j(i)}^{2}-1\right)^{2}}{n_{i}^{2}}-\sum_{j \neq k}^{n_{i}} \frac{\lambda_{1(i)}^{2}\left(z_{1 j(1)}^{2}-1\right)\left(z_{1 k(1)}^{2}-1\right)}{n_{i}^{2}\left(n_{i}-1\right)}\right\} \\
& +O_{p}\left(n_{\max }^{1 / 2} K \xi^{2}\right)+o_{p}\left\{(K \Delta)^{1 / 2}\right\}+o_{p}(K) .
\end{aligned}
$$

Here, it holds that

$$
\sum_{i=1}^{2}\left\{\sum_{j=1}^{n_{1}} \frac{\lambda_{1(i)}^{2}\left(z_{1 j(i)}^{2}-1\right)^{2}}{n_{i}}\right\}=K+o_{p}(K)
$$

under (A-iii) from the fact that $E\left\{\left(z_{1 j(i)}^{2}-1\right)^{2}\right\}=2$ for all $i, j$. Then, by noting that $\sum_{j \neq k}^{n_{i}} \lambda_{1(i)}^{2}\left(z_{1 j(1)}^{2}-\right.$ $1)\left(z_{1 k(1)}^{2}-1\right) /\left\{n_{i}^{2}\left(n_{i}-1\right)\right\}=o_{p}(K)$ for $i=1,2$, we can claim the result from (B.8).

Proofs of Proposition 2.1 and Theorem 2.1. Note that $E\left\{\left(z_{1 j(i)}^{2}-1\right)^{2}\right\}=2$ for all $i, j$, under (A-iii). By using Lyapunov's central limit theorem, it holds that as $m \rightarrow \infty$

$$
K^{-1 / 2}\left(\sum_{j=1}^{n_{1}} \frac{\lambda_{1(1)}\left(z_{1 j(1)}^{2}-1\right)}{n_{1}}-\sum_{k=1}^{n_{2}} \frac{\lambda_{1(2)}\left(z_{1 k(2)}^{2}-1\right)}{n_{2}}\right) \Rightarrow N(0,1)
$$

under (A-iii). Thus, from Lemmas 2.1 and A.1, we can claim the results.

Proof of Theorem 2.2. Assume (A-i) and (1.3). For the first result of Theorem 2.2, by noting that $\operatorname{tr}\left(\boldsymbol{\Sigma}_{1} \boldsymbol{\Sigma}_{2}\right) /\left(n_{1} n_{2}\right) \leq\left\{\operatorname{tr}\left(\boldsymbol{\Sigma}_{1}^{2}\right) \operatorname{tr}\left(\boldsymbol{\Sigma}_{2}^{2}\right) /\left(n_{1} n_{2}\right)^{2}\right\}^{1 / 2}=O\left(K^{2}\right)$ and $\operatorname{tr}\left[\left\{\boldsymbol{\Sigma}_{i}\left(\boldsymbol{\Sigma}_{1}-\boldsymbol{\Sigma}_{2}\right)\right\}^{2}\right] / n_{i} \leq \lambda_{1(i)}^{2} \Delta / n_{i}=$ $O(K \Delta)$, from (2.3), it holds that $\operatorname{Var}\left(U_{n_{1}, n_{2}}\right) / \Delta^{2}=o(1)$ as $m \rightarrow \infty$ under $K / \Delta=o(1)$ as $m \rightarrow \infty$. Then, by noting that $\widetilde{K} / K=1+o_{p}(1)$, it holds that $\widetilde{T}_{n_{1}, n_{2}}=(\Delta / K)\left\{1+o_{p}(1)\right\}+1 \rightarrow \infty$ in probability under $K / \Delta=o(1)$. Thus, we can claim the first result of Theorem 2.2. A.1.

For the second result of Theorem 2.2, it is obtained straightforwardly from (2.7) and Lemma

Proof of Corollary 2.1. Note that $\Delta / K \rightarrow \infty$ as $m \rightarrow \infty$ under (1.3) and either $\lim \sup _{d \rightarrow \infty}$ $\left|\boldsymbol{h}_{1(1)}^{T} \boldsymbol{h}_{1(2)}\right|<1$ or $\liminf _{d \rightarrow \infty}\left|\lambda_{1(1)} / \lambda_{1(2)}-1\right|>0$. Hence, we obtain the result from Theorem 2.2 . 
Proofs of Propositions 3.1, 3.2 and Theorem 4.1. From Lemmas A.1 to A.3, (3.3) and (3.5), we can claim the results.

Proof of Theorem 4.2. Assume (A-i) and (A-ii). We first consider the first result of Theorem 4.2 when $\lim \inf _{d \rightarrow \infty}\left(\lambda_{1(1)}-\lambda_{1(2)}\right)^{2} / \Delta_{\star}>0$. From Lemma A.1, it holds that as $m \rightarrow \infty$

$$
T_{\text {IYA }} \geq\left[\lambda_{1(1)}\left\{1+O_{p}\left(n_{1}^{-1 / 2}\right)\right\}-\lambda_{1(2)}\left\{1+O_{p}\left(n_{2}^{-1 / 2}\right)\right\}\right]^{2}\left\{1+o_{p}(1)\right\} / K .
$$

Thus, when $\liminf _{d \rightarrow \infty}\left|\lambda_{1(1)} / \lambda_{1(2)}-1\right|>0$, it follows that $T_{\text {IYA }} \rightarrow \infty$ in probability under $K / \Delta_{\star}=$ $o(1)$. When $\lambda_{1(1)} / \lambda_{1(2)}=1+o(1)$, it holds that $n_{\min }\left(\lambda_{1(1)} / \lambda_{1(2)}-1\right)^{2} \rightarrow \infty$ under $K / \Delta_{\star}=o(1)$. Hence, by noting that $\left[\lambda_{1(1)}\left\{1+O_{p}\left(n_{1}^{-1 / 2}\right)\right\}-\lambda_{1(2)}\left\{1+O_{p}\left(n_{2}^{-1 / 2}\right)\right\}\right]^{2}=\lambda_{1(2)}^{2}\left\{\lambda_{1(1)} / \lambda_{1(2)}-1+\right.$ $\left.O_{p}\left(n_{\text {min }}^{-1 / 2}\right)\right\}^{2}$, it holds that $T_{\text {IYA }} \rightarrow \infty$ in probability under $K / \Delta_{\star}=o(1)$ when $\lambda_{1(1)} / \lambda_{1(2)}=1+o(1)$.

Next, we consider the first result of Theorem 4.2 when $\lambda_{1(1)} / \lambda_{1(2)}=1+o(1)$ and

$$
\liminf _{d \rightarrow \infty} \lambda_{1(1)} \lambda_{1(2)} \xi^{2(1+\eta)} / \Delta_{\star}>0 .
$$

Note that $n_{\min } \xi^{2} \rightarrow \infty$ under $K / \Delta_{\star}=o(1)$. Then, from Lemma A.1, it holds that

$$
\begin{aligned}
T_{\text {IYA }} & \geq 2 \lambda_{1(1)} \lambda_{1(2)}\left\{\xi^{2}+O_{p}\left(\xi n_{\min }^{-1 / 2}\right)\right\}^{1+\eta+o_{p}(1)}\left\{1+o_{p}(1)\right\} / K \\
& =2 \lambda_{1(1)} \lambda_{1(2)} \xi^{2(1+\eta)+o_{p}(1)}\left\{1+o_{p}(1)\right\} / K \rightarrow \infty
\end{aligned}
$$

in probability under $K / \Delta_{\star}=o(1)$. Thus, we can claim the first result from (4.1).

For the second result of Theorem 4.2, from Lemmas A.2 and A.3, it holds that

$$
T_{\mathrm{IYA}} \geq \kappa_{\star} \delta_{\star}\left\{1+o_{p}(1)\right\}\left(\tilde{\lambda}_{1(1)}-\tilde{\lambda}_{1(2)}\right)^{2} / K .
$$

Note that $\left\{\tilde{\lambda}_{1(1)}-\tilde{\lambda}_{1(2)}-\left(\lambda_{1(1)}-\lambda_{1(2)}\right)\right\} / K^{1 / 2} \Rightarrow N(0,1)$ under (A-iii). Then, we can claim the second result.

Proof of Corollary 4.1. Note that $\Delta_{\star} / K \rightarrow \infty$ as $m \rightarrow \infty$ under (A-ii) and either

$$
\limsup _{d \rightarrow \infty}\left|\boldsymbol{h}_{1(1)}^{T} \boldsymbol{h}_{1(2)}\right|<1 \text { or } \liminf _{d \rightarrow \infty}\left|\lambda_{1(1)} / \lambda_{1(2)}-1\right|>0 .
$$

Hence, we can claim the result by using Theorem 4.2 .

Proof of Corollary 4.2. From Corollary 4.1, we consider the case when $\lambda_{1(1)} / \lambda_{1(2)}=1+o(1)$ as $d \rightarrow \infty$. Then, by noting that $\eta \rightarrow 0$ as $m \rightarrow \infty$ under (1.3), it holds that $K / \Delta_{\star}=O\left(n_{\text {min }}^{\beta-1}\right)=o(1)$ under $\liminf \operatorname{in}_{m \rightarrow \infty} n_{\min }^{\beta}\left(1-\left|\boldsymbol{h}_{1(1)}^{T} \boldsymbol{h}_{1(2)}\right|\right)>0$ for some constant $\beta \in(0,1)$. Hence, we can claim the result by using Theorem 4.2.

Proof of Lemma A.1. We assume (A-i) and (A-ii). Let $\boldsymbol{u}_{j(i)}=\left(z_{j 1(i)}, \ldots, z_{j n_{i}(i)}\right)^{T} /\left(n_{i}-1\right)^{1 / 2}$, $\boldsymbol{u}_{o j(i)}=\left(z_{j 1(i)}-\bar{z}_{j(i)}, \ldots, z_{j n_{i}(i)}-\bar{z}_{j(i)}\right)^{T} /\left(n_{i}-1\right)^{1 / 2}$ and $\dot{\boldsymbol{u}}_{o j(i)}=\left\|\boldsymbol{u}_{o j(i)}\right\|^{-1} \boldsymbol{u}_{o j(i)}$ for all $i, j$, where $\bar{z}_{j(i)}=\sum_{k=1}^{n_{i}} z_{j k(i)} / n_{i}$ and $\|\cdot\|$ denotes the Euclidean norm. Let $\sigma_{i}=\delta_{i}^{1 / 2} / \lambda_{1(i)}$ for $i=1,2$. We assume $\hat{\boldsymbol{u}}_{1(i)}^{T} \dot{\boldsymbol{u}}_{o 1(i)} \geq 0$ for $i=1,2$, without loss of generality. We first show that

$$
\begin{aligned}
& \left(\hat{\lambda}_{1(i)}-\kappa_{i}\right) / \lambda_{1(i)}=\left\|\boldsymbol{u}_{o 1(i)}\right\|^{2}+O_{p}\left(\sigma_{i} n_{i}^{-1}\right) \text { and } \\
& \hat{\boldsymbol{u}}_{1(i)}^{T} \dot{\boldsymbol{u}}_{o 1(i)}=1+O_{p}\left(\sigma_{i}^{2} n_{i}^{-1}\right) \text { as } m \rightarrow \infty \text { for } i=1,2 .
\end{aligned}
$$


Throughout the proof of (B.10), we omit the subscript with regard to the population. Let $\boldsymbol{\zeta}$ be an arbitrary unit random $n$-dimensional vector. Let us write that $\boldsymbol{U}_{1}=\lambda_{1} \boldsymbol{u}_{o 1} \boldsymbol{u}_{o 1}^{T}, \boldsymbol{U}_{2}=\sum_{s=2}^{p} \lambda_{s} \boldsymbol{u}_{s} \boldsymbol{u}_{s}^{T}$ and $\boldsymbol{U}_{22}=\boldsymbol{U}_{2}-\kappa \boldsymbol{I}_{n} /(n-1)$. Let $\boldsymbol{P}_{n}=\boldsymbol{I}_{n}-\mathbf{1}_{n} \mathbf{1}_{n}^{T} / n$, where $\mathbf{1}_{n}=(1, \ldots, 1)^{T}$. Note that $\boldsymbol{S}_{D}=\boldsymbol{U}_{1}+\boldsymbol{P}_{n} \boldsymbol{U}_{2} \boldsymbol{P}_{n}$. Also, note that $\boldsymbol{P}_{n} \hat{\boldsymbol{u}}_{1}=\hat{\boldsymbol{u}}_{1}$ and $\hat{\lambda}_{1}=\hat{\boldsymbol{u}}_{1}^{T} \boldsymbol{S}_{D} \hat{\boldsymbol{u}}_{1}=\hat{\boldsymbol{u}}_{1}^{T}\left(\boldsymbol{U}_{1}+\boldsymbol{U}_{2}\right) \hat{\boldsymbol{u}}_{1}$ when $\hat{\lambda}_{1}>0$. Here, under (A-ii), there exists a positive fixed integer $g(\geq 2)$ such that $\lambda_{g} / \lambda_{1}=o(1)$ as $d \rightarrow \infty$. Let $\boldsymbol{\Sigma}_{g}=\sum_{s=g}^{d} \lambda_{s} \boldsymbol{h}_{s} \boldsymbol{h}_{s}^{T}$. Note that

$$
\operatorname{tr}\left(\boldsymbol{\Sigma}_{g}^{4}\right) / \lambda_{1}^{4} \leq\left(\lambda_{g} / \lambda_{1}\right)^{2} \operatorname{tr}\left(\boldsymbol{\Sigma}_{g}^{2}\right) / \lambda_{1}^{2}=o(1)
$$

under (A-ii). Then, from Lemma 3 in Yata and Aoshima [5], we have that

$$
\frac{\boldsymbol{\zeta}^{T} \boldsymbol{U}_{22} \boldsymbol{\zeta}}{\lambda_{1}}=\sum_{s=2}^{g-1} \frac{\lambda_{s}}{\lambda_{1}}\left(\boldsymbol{\zeta}^{T} \boldsymbol{u}_{s}\right)^{2}+o_{p}(1) .
$$

Similar to the proofs of Lemmas 3 and 4 in Yata and Aoshima [4], we have that

$$
\boldsymbol{u}_{1}^{T} \boldsymbol{U}_{22} \boldsymbol{u}_{1}=O_{p}\left(\delta^{1 / 2} / n\right) \text { and } \boldsymbol{u}_{1}^{T} \boldsymbol{U}_{22} \boldsymbol{\zeta}=O_{p}\left(\delta^{1 / 2} / n^{1 / 2}\right) .
$$

There exist a random variable $\varepsilon \in[0,1]$ and a unit random $n$-dimensional vector $\boldsymbol{v}$ such that $\hat{\boldsymbol{u}}_{1}=\left(1-\varepsilon^{2}\right)^{1 / 2} \dot{\boldsymbol{u}}_{o 1}+\varepsilon \boldsymbol{v}$ and $\dot{\boldsymbol{u}}_{o 1}^{T} \boldsymbol{v}=0$. Then, from (B.12) and (B.13), we have that

$$
\begin{aligned}
& \frac{\hat{\lambda}_{1}-\kappa /(n-1)}{\lambda_{1}}=\hat{\boldsymbol{u}}_{1}^{T}\left(\boldsymbol{U}_{1}+\lambda_{1}^{-1} \boldsymbol{U}_{22}\right) \hat{\boldsymbol{u}}_{1} \\
& =\left\|\boldsymbol{u}_{o 1}\right\|^{2}+\max _{\varepsilon}\left\{-\varepsilon^{2}\left\|\boldsymbol{u}_{o 1}\right\|^{2}\left\{1+o_{p}(1)\right\}+O_{p}\left(\varepsilon \sigma / n^{1 / 2}\right)+\varepsilon^{2} \sum_{s=2}^{g-1} \frac{\lambda_{s}}{\lambda_{1}}\left(\boldsymbol{v}^{T} \boldsymbol{u}_{s}\right)^{2}\right\} \\
& \quad+O_{p}(\sigma / n) .
\end{aligned}
$$

It follows that

$$
\begin{aligned}
& \max _{\varepsilon}\left\{-\varepsilon^{2}\left\|\boldsymbol{u}_{o 1}\right\|^{2}\left\{1+o_{p}(1)\right\}+O_{p}\left(\frac{\varepsilon \sigma}{n^{1 / 2}}\right)+\varepsilon^{2} \sum_{s=2}^{g-1} \frac{\lambda_{s}}{\lambda_{1}}\left(\boldsymbol{v}^{T} \boldsymbol{u}_{s}\right)^{2}\right\} \\
& \leq \max _{\varepsilon}\left\{-\varepsilon^{2}\left\{1+o_{p}(1)\right\}+O_{p}\left(\frac{\varepsilon \sigma}{n^{1 / 2}}\right)+\varepsilon^{2} \frac{\lambda_{2}}{\lambda_{1}}\left\{1+o_{p}(1)\right\}\right\}=O_{p}\left(\sigma^{2} / n\right), \\
& \text { so that } \varepsilon=O_{p}\left(\sigma / n^{1 / 2}\right) .
\end{aligned}
$$

Hence, we can conclude the results in (B.10).

Next, we consider the proofs of the results in Lemma A.1. Note that

$$
\operatorname{tr}\left(\boldsymbol{S}_{i D}\right)=\lambda_{1(i)}\left\|\boldsymbol{u}_{o 1(i)}\right\|^{2}+\kappa_{i}+o_{p}\left(\delta_{i}^{1 / 2}\right)
$$

from the fact that $\operatorname{Var}\left\{\operatorname{tr}\left(\boldsymbol{S}_{i D}\right)-\lambda_{1(i)}\left\|\boldsymbol{u}_{o 1(i)}\right\|^{2}\right\}=\operatorname{Var}\left(\sum_{s=2}^{d} \lambda_{s(i)}\left\|\boldsymbol{u}_{o s(i)}\right\|^{2}\right)=O\left(\delta_{i} / n_{i}\right)$. Then, from (B.10) we can conclude the first result in Lemma A.1. We assume $\boldsymbol{h}_{1(i)}^{T} \tilde{\boldsymbol{h}}_{1(i)} \geq 0, i=1$, 2 , and $\boldsymbol{h}_{1(1)}^{T} \boldsymbol{h}_{1(2)} \geq 0$ without loss of generality. We note that $\left(\boldsymbol{X}_{i}-\overline{\boldsymbol{X}}_{i}\right) \hat{\boldsymbol{u}}_{1(i)}=\boldsymbol{X}_{i} \hat{\boldsymbol{u}}_{1(i)}$ and $\hat{\boldsymbol{u}}_{1(i)}^{T} \boldsymbol{u}_{o 1(i)}=$ $\hat{\boldsymbol{u}}_{1(i)}^{T} \boldsymbol{u}_{1(i)}$ when $\hat{\lambda}_{1(i)}>0$. Let $h_{s t}=\boldsymbol{h}_{s(1)}^{T} \boldsymbol{h}_{t(2)}$ for all $s, t$. From (B.10) and the first result in Lemma A.1 we have that

$$
\begin{aligned}
\tilde{\boldsymbol{h}}_{1(1)}^{T} \boldsymbol{h}_{1(2)} & =\frac{\hat{\boldsymbol{u}}_{1(1)}^{T} \boldsymbol{X}_{1}^{T} \boldsymbol{h}_{1(2)}}{\left\{\left(n_{i}-1\right) \tilde{\lambda}_{1(1)}\right\}^{1 / 2}}=\frac{\hat{\boldsymbol{u}}_{1(1)}^{T} \sum_{s=1}^{d} \lambda_{s(1)}^{1 / 2} h_{s 1} \boldsymbol{u}_{s(1)}}{\tilde{\lambda}_{1(1)}^{1 / 2}} \\
& =h_{11}+\frac{\hat{\boldsymbol{u}}_{1(1)}^{T} \sum_{s=2}^{d} \lambda_{s(1)}^{1 / 2} h_{s 1} \boldsymbol{u}_{s(1)}}{\lambda_{1(1)}^{1 / 2}\left\{1+o_{p}(1)\right\}}+O_{p}\left(\sigma_{1} / n_{1}\right) .
\end{aligned}
$$


Note that $E\left\{\left(\boldsymbol{u}_{1(1)}^{T} \sum_{s=2}^{d} \lambda_{s(1)}^{1 / 2} h_{s 1} \boldsymbol{u}_{s(1)}\right)^{2}\right\}=O\left(\boldsymbol{h}_{1(2)}^{T} \boldsymbol{\Sigma}_{1 \star} \boldsymbol{h}_{1(2)} / n_{1}\right)$ and $E\left(\left\|\sum_{s=2}^{d} \lambda_{s(1)}^{1 / 2} h_{s 1} \boldsymbol{u}_{s(1)}\right\|^{2}\right)=$ $O\left(\boldsymbol{h}_{1(2)}^{T} \boldsymbol{\Sigma}_{1 \star} \boldsymbol{h}_{1(2)}\right)$. Here, there exists a $d$-dimensional unit vector $\boldsymbol{f}$ such that $\boldsymbol{h}_{1(2)}=\left(1-\xi^{2}\right)^{1 / 2} \boldsymbol{h}_{1(1)}+$ $\xi \boldsymbol{f}$ and $\boldsymbol{h}_{1(1)}^{T} \boldsymbol{f}=0$. Then, it holds that $\boldsymbol{h}_{1(2)}^{T} \boldsymbol{\Sigma}_{1 \star} \boldsymbol{h}_{1(2)}=O\left(\xi^{2} \lambda_{2(1)}\right)=O\left(\xi^{2} \delta_{1}^{1 / 2}\right)$. Thus, by using Markov's inequality, it follows from (B.14) and (B.15) that

$$
\begin{aligned}
\tilde{\boldsymbol{h}}_{1(1)}^{T} \boldsymbol{h}_{1(2)}-h_{11} & =O_{p}\left\{\left(\frac{\boldsymbol{h}_{1(2)}^{T} \boldsymbol{\Sigma}_{1 \star} \boldsymbol{h}_{1(2)}}{n_{1} \lambda_{1(1)}}\right)^{1 / 2}\right\}+O_{p}\left(\sigma_{1} / n_{1}\right) \\
& =O_{p}\left(\xi \sigma_{1}^{1 / 2} / n_{1}^{1 / 2}\right)+O_{p}\left(\sigma_{1} / n_{1}\right) .
\end{aligned}
$$

As for $\boldsymbol{h}_{1(1)}^{T} \tilde{\boldsymbol{h}}_{1(2)}-\boldsymbol{h}_{1(1)}^{T} \boldsymbol{h}_{1(2)}$, we obtain the result similarly. It follows that

$$
\begin{aligned}
& \frac{\hat{\boldsymbol{u}}_{1(1)}^{T}\left(\sum_{s=1}^{d} \lambda_{s(1)}^{1 / 2} \lambda_{1(2)}^{1 / 2} h_{s 1} \boldsymbol{u}_{s(1)} \boldsymbol{u}_{1(2)}^{T}\right) \hat{\boldsymbol{u}}_{1(2)}}{\tilde{\lambda}_{1(1)}^{1 / 2} \tilde{\lambda}_{1(2)}^{1 / 2}}=\frac{\hat{\boldsymbol{u}}_{1(1)}^{T} \sum_{s=1}^{d} \lambda_{s(1)}^{1 / 2} h_{s 1} \boldsymbol{u}_{s(1)}}{\tilde{\lambda}_{1(1)}^{1 / 2}}\left\{1+O_{p}\left(\sigma_{2} / n_{2}\right)\right\} \\
& =\tilde{\boldsymbol{h}}_{1(1)}^{T} \boldsymbol{h}_{1(2)}+O_{p}\left(\sigma_{2} / n_{2}\right) \quad \text { and } \\
& \frac{\hat{\boldsymbol{u}}_{1(1)}^{T}\left(\sum_{s=1}^{d} \lambda_{1(1)}^{1 / 2} \lambda_{s(2)}^{1 / 2} h_{1 s} \boldsymbol{u}_{1(1)} \boldsymbol{u}_{s(2)}^{T}\right) \hat{\boldsymbol{u}}_{1(2)}}{\tilde{\lambda}_{1(1)}^{1 / 2} \tilde{\lambda}_{1(2)}^{1 / 2}}=\boldsymbol{h}_{1(1)}^{T} \tilde{\boldsymbol{h}}_{1(2)}+O_{p}\left(\sigma_{1} / n_{1}\right)
\end{aligned}
$$

from the equation (S6.23) in the supplementary material of Aoshima and Yata [1], (B.10) and the first result in Lemma A.1. Then, from the equation (S6.26) in Aoshima and Yata [1], we have that

$$
\begin{aligned}
& \tilde{\boldsymbol{h}}_{1(1)}^{T} \tilde{\boldsymbol{h}}_{1(2)}=\frac{\hat{\boldsymbol{u}}_{1(1)}^{T}\left(\sum_{s, s^{\prime}}^{d} \lambda_{s(1)}^{1 / 2} \lambda_{s^{\prime}(2)}^{1 / 2} h_{s s^{\prime}} \boldsymbol{u}_{s(1)} \boldsymbol{u}_{s^{\prime}(2)}^{T}\right) \hat{\boldsymbol{u}}_{1(2)}}{\tilde{\lambda}_{1(1)}^{1 / 2} \tilde{\lambda}_{1(2)}^{1 / 2}} \\
& =\tilde{\boldsymbol{h}}_{1(1)}^{T} \boldsymbol{h}_{1(2)}+\boldsymbol{h}_{1(1)}^{T} \tilde{\boldsymbol{h}}_{1(2)}-h_{11} \frac{\hat{\boldsymbol{u}}_{1(1)}^{T}\left(\lambda_{1(1)}^{1 / 2} \lambda_{1(2)}^{1 / 2} \boldsymbol{u}_{1(1)} \boldsymbol{u}_{1(2)}^{T}\right) \hat{\boldsymbol{u}}_{1(2)}}{\tilde{\lambda}_{1(1)}^{1 / 2} \tilde{\lambda}_{1(2)}^{1 / 2}}+O_{p}\left(\sigma_{1} / n_{1}+\sigma_{2} / n_{2}\right) \\
& =\tilde{\boldsymbol{h}}_{1(1)}^{T} \boldsymbol{h}_{1(2)}+\boldsymbol{h}_{1(1)}^{T} \tilde{\boldsymbol{h}}_{1(2)}-h_{11}+O_{p}\left(\sigma_{1} / n_{1}+\sigma_{2} / n_{2}\right) .
\end{aligned}
$$

Then, from (B.16) we have that

$$
\tilde{\boldsymbol{h}}_{1(1)}^{T} \tilde{\boldsymbol{h}}_{1(2)}-h_{11}=O_{p}\left\{\xi\left(\sigma_{1}^{1 / 2} / n_{1}^{1 / 2}+\sigma_{2}^{1 / 2} / n_{2}^{1 / 2}\right)+\sigma_{1} / n_{1}+\sigma_{2} / n_{2}\right\} .
$$

Hence, we can conclude the second result in Lemma A.1.

For the proof of the third result in Lemma A.1, the result is obtained straightforwardly by using the first result in Lemma A.1.

Proof of Lemma A.2. When $\lambda_{2(i)}^{2} / \delta_{i} \rightarrow 0$ as $d \rightarrow \infty$, we can claim the result by using Lemma S2.1 in Aoshima and Yata [1]. Thus, we consider the case when $\liminf _{d \rightarrow \infty} \lambda_{2(i)}^{2} / \delta_{i}>0$. Similar to (B.11), there exists a positive fixed integer $h(\geq 2)$ such that $\lambda_{h+1(i)} / \lambda_{h(i)}=o(1)$ and $\liminf _{d \rightarrow \infty} \lambda_{h(i)}^{2} / \delta_{i}>$ 0 . Note that $\sum_{s=h+1}^{d} \lambda_{s(i)}^{4} / \lambda_{h(i)}^{4} \leq\left(\lambda_{h+1(i)} / \lambda_{h(i)}\right)^{2} \delta_{i} / \lambda_{h(i)}^{2}=o(1)$. Then, from the equation (A.10) and Lemma 12 in Yata and Aoshima [5], we have that as $m \rightarrow \infty$

$$
\frac{\hat{\delta}_{i}}{\delta_{i}}=\frac{\sum_{s=h+1}^{d} \lambda_{s(i)}^{2}+\sum_{s=2}^{h} \hat{\lambda}_{s(i)}^{2}}{\delta_{i}}+o_{p}(1)=1+o_{p}(1)
$$


under (A-i) and (A-ii). It concludes the result.

Proof of Lemma A.3. When (1.3) is met, we can claim the result by using Lemma 2.1 in Ishii et al. [2]. Thus, we consider the case when

$$
\liminf _{d \rightarrow \infty} \lambda_{1(i)}^{2} / \delta_{i}>0 \text { and } \limsup _{d \rightarrow \infty} \lambda_{1(i)}^{2} / \delta_{i}<\infty .
$$

We have that $\operatorname{tr}\left(\boldsymbol{S}_{i D}\right)=\operatorname{tr}\left(\boldsymbol{\Sigma}_{i}\right)+o_{p}\left(\delta_{i}^{1 / 2}\right)$ under $(\mathrm{A}-\mathrm{i})$ since $\operatorname{Var}\left\{\operatorname{tr}\left(\boldsymbol{S}_{i D}\right)\right\}=O\left\{\operatorname{tr}\left(\boldsymbol{\Sigma}_{i}^{2}\right) / n_{i}\right\}$. By noting that $\tilde{\lambda}_{1(i)}=\lambda_{1(i)}+o_{p}\left(\delta_{i}^{1 / 2}\right)$ under (A-i) and (A-ii) from Lemma A.1, it holds that $\hat{\kappa}_{i}=$ $\kappa_{i}+o_{p}\left(\delta_{i}^{1 / 2}\right)$ under $(\mathrm{A}-\mathrm{i})$. Since $\delta_{i} / \kappa_{i}^{2} \leq 1$, we can claim the result.

\section{Appendix C. A numerical result under the NSSE model}

In this section, we give an additional simulation result for Section 5 in Ishii et al. [3]. As for the covariance matrices in (5.3), we considered the alternative hypothesis given in (iii) or (iv). We followed the same settings as in Fig. 3 of Ishii et al. [3]. We set

$$
\alpha_{1}=2 / 5 .
$$

Then, the NSSE condition (1.2) is met. We considered the non-Gaussian distribution (II) given in Section 5 of Ishii et al. [3]. Similar to Figs. 2 and 3 in Ishii et al. [3], we calculated $\bar{\alpha}$ and $1-\bar{\beta}$ by 2000 replications and plotted the results in Fig. C.1.

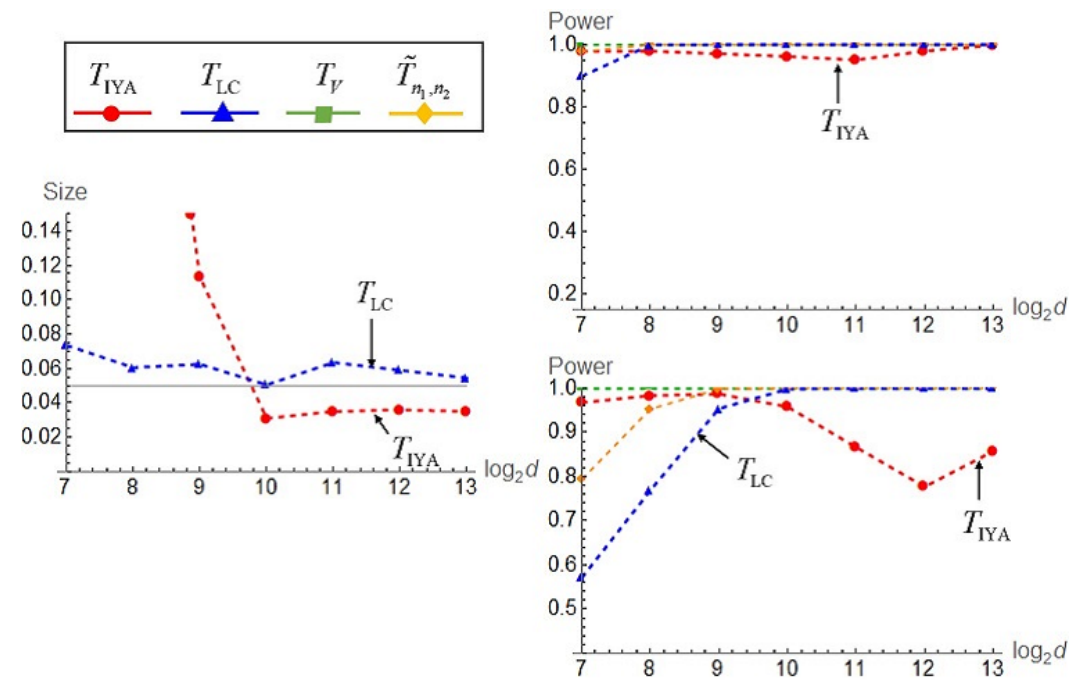

Figure C.1: The performance of the test statistics, $T_{\mathrm{LC}}, \widetilde{T}_{n_{1}, n_{2}}, T_{V}$ and $T_{\mathrm{IYA}}$, under the NSSE model (1.2). We set $\left(n_{1}, n_{2}\right)=\left(3\left\lceil d^{1 / 2}\right\rceil, 4\left\lceil d^{1 / 2}\right\rceil\right)$ and $d=2^{s}$ for $s=7, \ldots, 13$. The value of $\bar{\alpha}$ is denoted by the dashed line in the left panel. The value of $1-\bar{\beta}$ is denoted by the dashed line in the upper right panel for (iii) and in the lower right panel for (iv). In the left panel, the values of $\bar{\alpha}$ for $\widetilde{T}_{n_{1}, n_{2}}$ and $T_{V}$ were too high to describe.

One can observe that $T_{\mathrm{LC}}$ gave a preferable performance for (C.1) because $T_{\mathrm{LC}}$ was constructed under the NSSE model (1.2). On the other hand, $T_{\mathrm{IYA}}$ gave adequate performances as $d$ and $n_{i} \mathrm{~S}$ increase even though the NSSE condition (1.2) is met. This is probably because $\tilde{\lambda}_{1(i)}$ has a consistency property and asymptotic normality even when (1.2) is met. See Section 4 in Yata and Aoshima [5] for the details. 
As concluded in Section 7 of Ishii et al. [3], we recommend using a suitable test procedure which fits the eigenstructure.

\section{References}

[1] Aoshima, M., Yata, K., Two-sample tests for high-dimension, strongly spiked eigenvalue models. Stat. Sin. 28 (2018), 43-62.

[2] Ishii, A., Yata, K., Aoshima, M., Asymptotic properties of the first principal component and equality tests of covariance matrices in high-dimension, low-sample-size context. J. Stat. Plan. Inference 170 (2016), 186-199.

[3] Ishii, A., Yata, K., Aoshima, M., Equality tests of high-dimensional covariance matrices under the strongly spiked eigenvalue model.

[4] Yata, K., Aoshima, M., Effective PCA for high-dimension, low-sample-size data with noise reduction via geometric representations. J. Multivariate Anal. 105 (2012), 193-215.

[5] Yata, K., Aoshima, M., PCA consistency for the power spiked model in high-dimensional settings. J. Multivariate Anal. 122 (2013), 334-354. 